\title{
cFos ensembles in the dentate gyrus rapidly segregate over time and do not form a stable map of space
}

\author{
Paul J. Lamothe-Molina ${ }^{1 \#}$, Andreas Franzelin ${ }^{1}$, Lea Auksutat ${ }^{2}$, Laura Laprell ${ }^{1}$, Joachim
}

Alhbeck $^{3}$, Matthias Kneussel ${ }^{4}$, Andreas K. Engel ${ }^{3}$, Fabio Morellini ${ }^{2 *}$, Thomas G. Oertner ${ }^{1 * \#}$

${ }^{1}$ Institute for Synaptic Physiology, Center for Molecular Neurobiology (ZMNH), University Medical Center Hamburg-Eppendorf, Hamburg, Germany

${ }^{2}$ Research Group Behavioral Biology, Center for Molecular Neurobiology (ZMNH), University Medical Center Hamburg-Eppendorf, Hamburg, Germany

${ }^{3}$ Institute of Neurophysiology and Pathophysiology, Center for Experimental Medicine (ZEM), University Medical Center Hamburg-Eppendorf, Hamburg, Germany

${ }^{4}$ Institute for Molecular Neurogenetics, Center for Molecular Neurobiology (ZMNH), University Medical Center Hamburg-Eppendorf, Hamburg, Germany

*equal contribution

\#corresponding authors: thomas.oertner@zmnh.uni-hamburg.de

paul.lamothe@zmnh.uni-hamburg.de

\section{Summary}

Mice require several days of training to master the water maze, a spatial memory task for rodents. The hippocampus plays a key role in the formation of spatial and episodic memories, a process that involves the activation of immediate-early genes such as cFos. We trained cFos-reporter mice in the water maze, expecting that consistent spatial behavior would be reflected by consistent cFos patterns across training episodes. Even after extensive training, however, different sets of dentate gyrus (DG) granule cells were activated every day. Suppressing activity in the original encoding ensemble helped mice to learn a novel platform position (reversal learning). Our results suggest that even in a constant environment, cFos+ ensembles in the dorsal DG segregate as a function of time, but become partially reactivated when animals try to access memories of past events. 


\section{Introduction}

An accurate memory of past events and their outcomes is of high adaptive value for any animal, as past experience is a useful guide for decision making. The hippocampus is famous for its processing of spatial information (Bird \& Burgess, 2008; Morellini, 2013), but it also processes other types of sensory input and is capable of grouping events together in time to form episodic memories (Aronov et al., 2017; Eichenbaum, 2014; Mau et al., 2018). How time is represented in the hippocampus is still incompletely understood. Many genes affecting synaptic plasticity undergo pronounced circadian oscillations, changing the rules of synaptic plasticity depending on the time of day (McCauley et al., 2019). Theoretical and empirical work suggests that the dentate gyrus (DG) actively reduces the overlap of activity patterns coming from entorhinal cortex (Chavlis \& Poirazi, 2017; Deng et al., 2013; Leutgeb et al., 2007). In vivo electrophysiology and calcium imaging experiments revealed that the majority of GCs are silent, and of the active cells, just a small fraction show spatial tuning ("place cells") (Danielson et al., 2016, 2017; GoodSmith et al., 2017, 2019; Hainmueller \& Bartos, 2018; Senzai \& Buzsáki, 2017; van Dijk \& Fenton, 2018). Spatially-tuned GCs provide a stable, albeit coarse representation of the global environment across time. These findings highlight an obvious design conflict: While a perfect 'episode encoder' should avoid using the same neurons on consecutive days, accurate place coding, at least in the sense of labeled lines, requires consistent activation of specific neurons to signal the animal's position in a given environment. In view of this conundrum, we set out to study the impact of time and space on the activity of granule cells in dorsal DG.

To monitor and manipulate neuronal activity in freely behaving animals, reporter mice have been developed that use immediate-early genes (Denny et al., 2014; Reijmers et al., 2007) (cFos, Arc) to drive the expression of fluorescent proteins and optogenetic actuators (Josselyn \& Tonegawa, 2020). Fear conditioning experiments with activity-dependent expression of optogenetic silencing tools suggest that recall of a fearful episode requires reactivation of the original encoding ensemble (Denny et al., 2014; Lacagnina et al., 2019; Trouche et al., 2016). Vice versa, artificial reactivation (Lacagnina et al., 2019; Liu et al., 2012; Perusini et al., 2017; Ramirez et al., 2013) of the encoding ensemble has been shown to reinstate a fearful state, suggesting that memories can indeed be activated by specific subsets of hippocampal neurons. In these experiments, freezing was used as a proxy to assess the emotional state of the animal. However, due to the one-dimensional read-out, it is not possible to distinguish the precise recall of the conditioning episode from a general state of heightened anxiety.

We were interested in how the activity of old and new ensembles in DG would affect decision making in situations where the animal has a large number of possible choices. TetTag mice allow comparing activity-dependent expression of cFos at two different time points that can be several days apart (Khalaf et al., 2018; Ramirez et al., 2013). To be able to manipulate activity in freely behaving animals, we injected a virus encoding the chloride-conducting channelrhodopsin iChloC (Wietek et al., 2015) for light-controlled neuronal inhibition. We adapted the classic Morris water maze for mice tethered with thin optical fibers, using a helium balloon to compensate for the weight of the implant. 
As the expression of the inhibitory tool was restricted to the neurons active in the first tagging window, we were able to selectively silence this ensemble during probe trials or reversal training.

To our surprise, optogenetic inhibition had either no effect or even improved performance in the water maze. This finding was explained by the low cFos ensemble reactivation (overlap) at different stages of water maze training. Our results strongly support the proposed function of the DG in pattern separation and suggest that under conditions of high interference, reactivation of old ensembles can hinder the formation of new spatial memories.

\section{Results}

\section{Stable expression of optogenetic tools in active neurons for 6 days in TetTag mice}

To tag neuronal activity at two different time points, we bilaterally injected the DG of TetTag mice with AAV9-TRE-iChloC-mKate (Figure. 1a). Neuronal activity in TetTag mice activates the expression of two transgenes via the cFos promoter: 1) tetracycline transactivator (tTA) and 2) a short half-life, nuclear-localized, enhanced green fluorescent protein (shEGFP). In the absence of doxycycline (offDox), tTA binds to the tTA-response element (TRE) from the virally delivered gene, resulting in permanent mKate tagging of neurons active in the off-Dox time window (magenta). Green nuclear fluorescence (shEGFP) identifies neurons that were active in the hours before the animal was sacrificed (Figure 1b). In calibration experiments on slice cultures from TetTag mice, we found that shEGFP fluorescence peaked $3.5 \mathrm{~h}$ after high $\mathrm{K}^{+}$stimulation (Figure S1), suggesting that green-labeled neurons were active 2-4 $\mathrm{h}$ before sacrificing the animal. Cells that were active off-Dox and again in the hours before euthanasia could be readily identified ex-vivo by their magenta (mKate) membrane and green (shEGFP) nucleus, both of which were amplified by indirect immunofluorescence (Figure 1b).

To evaluate the ensemble size and stability of iChloC-mKate across time, injected TetTag mice were taken off-Dox two weeks after AAV injection. The bedding in the home cage was changed to prevent mice from eating any Dox food crumbles. Twenty-four hours later, mice were either kept undisturbed in their home cage or trained ( 6 trials, see Methods) in the water maze (WM). Homecaged mice were sacrificed on the same day $(n=3$ mice) or 1 day later ( $n=4$ mice). WM-trained mice were sacrificed 1 day later $(n=4)$ or 6 days later $(n=7$ mice) (Fig $1 c)$. To compare cFos tagging efficiencies between virally transfected (iChloC-mKate) and transgenic (shEGFP), mice were trained in the WM on the day of euthanasia (identical number of trials). Mice that were always on-Dox (home caged) showed no mKate expression. Home-caged mice sacrificed on tagging day ( $0 \mathrm{~d}$ ) showed significantly fewer mKate+ cells than mice sacrificed on the following day, probably due to the slow maturation of iChloC-mKate. Surprisingly, WM-trained mice showed similar ensemble sizes as home cage-exploration mice, probably because the bedding change triggered cFos expression as seen when novel objects are introduced in their home cage (Bernstein et al., 2019). Because WM learning requires more than one training day to develop a consistent allothetic navigation strategy, it was 
bioRxiv preprint doi: https://doi.org/10.1101/2020.08.29.273391; this version posted August 31, 2020. The copyright holder for this preprint (which was not certified by peer review) is the author/funder. All rights reserved. No reuse allowed without permission.

crucial that the cFos neuronal ensemble tagging lasted for at least six days. We found similar ensemble sizes in WM-trained mice that were sacrificed 1 or 6 days later (Figure $1 \mathrm{~d}, \mathrm{e}$ ).

a

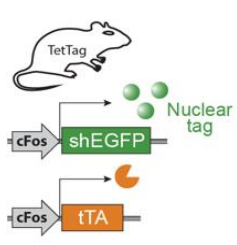

C

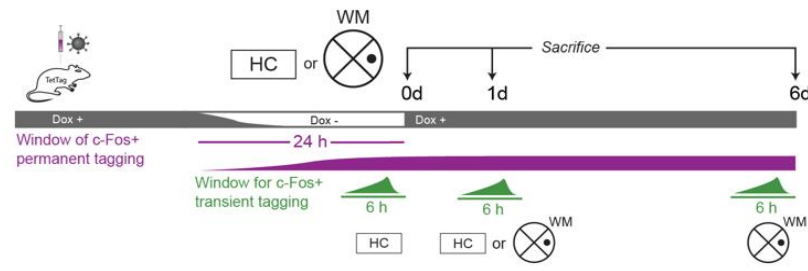

b
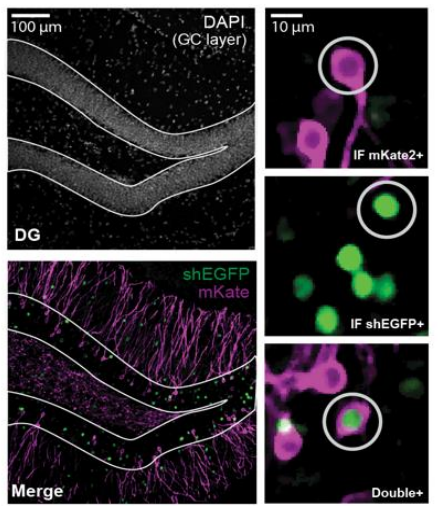

d

Dox +

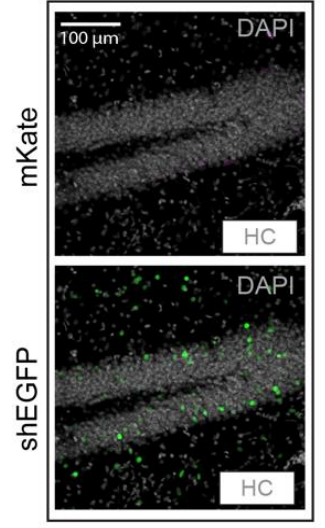

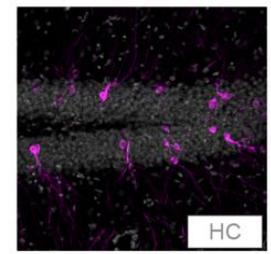

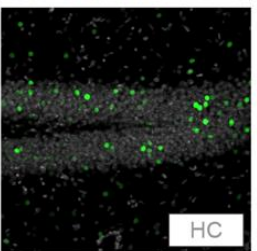

Od

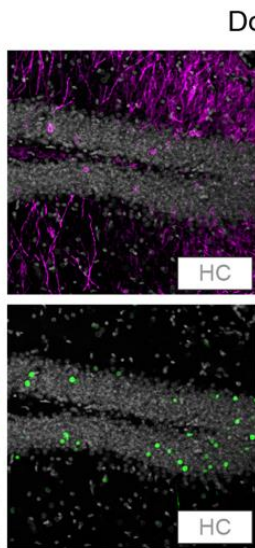

$1 d$

Dox -

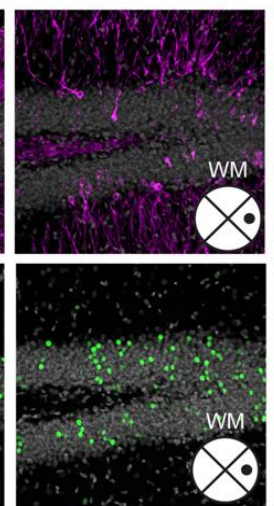

$1 d$

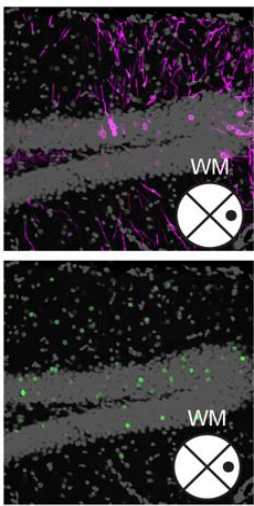

$6 d$

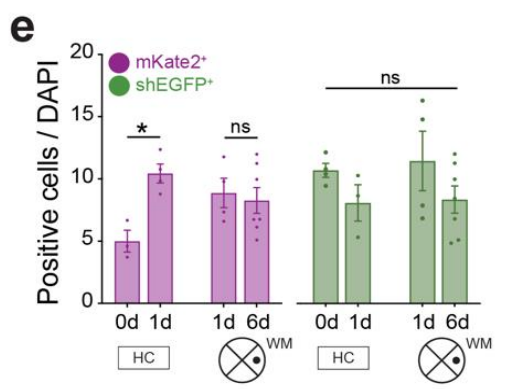

Figure 1. General experimental design for tagging two c-Fos ${ }^{+}$neuronal ensembles suitable for WM training. a, TetTag mice containing two cFos-driven transgenes: 1) tetracycline trans-activator (tTA) and 2) nuclear-localized, short-life enhanced green fluorescent protein (shEGFP). To tag (and manipulate) cFos + cells, TetTag mice are injected bilaterally in DG with an AAV containing the improved version of a chloride-conducting channel rhodopsin (iChloC) under the control of a tTA response element (TRE). Doxycycline (black triangle) prevents tTA from activating TRE-iChloC-mKate expression. When Dox is removed, active GCs express TRE-iChloC-mKate. shEGFP expression is independent of Dox. b, Experiment timeline. TetTag mice were injected with AAVs in DG on-Dox. Two weeks later, Dox was removed for $24 \mathrm{~h}$ (Dox-off). Some mice were in their home cage $(\mathrm{HC})$ and sacrificed $24 \mathrm{~h}$ after Dox removal $(0 \mathrm{~d}, \mathrm{n}=3)$ or $48 \mathrm{~h}$ after Dox removal $(1 \mathrm{~d}$, $n=3)$. A different cohort of mice experienced water maze (WM) training off-Dox. After training, mice were put back on Dox and were either sacrificed $48 \mathrm{~h}$ after Dox removal $(1 \mathrm{~d}, \mathrm{n}=4)$ or 6 days after $(6 \mathrm{~d}, \mathrm{n}=7)$. c, Confocal images showing DG. All tagged-GCs were counted within the limits of the GC layer (DAPI). iChloC-mKate expresses on GC membrane (pseudocoloured in magenta) while shEGFP (green) is only in the nuclei. Overlapping cells show magenta membrane and green nuclei. d, Confocal image set showing both GCs ensembles for all conditions. Dox prevents iChloC-mKate expression. f, Ensemble size (mKate+/DAPI and shEGFP+/DAPI). mKate+ ensemble size is significantly lower in the $0 \mathrm{~d}$ than $1 \mathrm{~d}\left({ }^{*}, \mathrm{p}=0.03\right.$, Brown-Forsythe ANOVA, Dunnet multiple comparisons Od vs. $\left.1 \mathrm{~d}\right)$. mKate+ ensemble size 
was similar regardless of experience (HC or WM) 1d after Dox removal. mKate ensemble size was not decreased 6 days after tagging. shEGFP ensemble size was similar regardless of experience (HC or WM) or day of sacrifice.

\section{Optogenetic silencing of cFos+ DG ensembles in the water maze}

In fear conditioning experiments, cFos+ ensembles are often regarded as the trace of a particular memory (Josselyn \& Tonegawa, 2020). To test whether the recall of a spatial memory requires reactivation of the same set of neurons that were cFos+ during the first encoding day, we tagged cFos+ cells in DG with the light-activated chloride channel iChloC (Wietek et al., 2015) ( $n=16$ mice) (Figure 2a). Compared to light-driven pumps, this channel requires lower light intensities for efficient inhibition (Wiegert et al., 2017), allowing the inhibition of a larger volume of tissue from a single light fiber. In the absence of light, membrane properties of iChloC-expressing GCs were indistinguishable from those reported in the literature (NeuroElectro :: Dentate Gyrus Granule Cell, n.d.) (Figure S2). Blue light $(473 \mathrm{~nm}$ ) activated iChloC, preventing action potential generation (Figure S2). For inhibition of tagged GCs in vivo, blue light pulses $(1 \mathrm{~Hz})$ were delivered bilaterally through a two-ferrule cannula targeting the hippocampal sulcus directly above DG (Figure 2a). To aid the swimming of tethered mice, we overcompensated the weight of the implant and the two optical fibers with a helium balloon, resulting in a slight pull-up force of $0.07 \mathrm{~N}$ (Figure 2b). Under these conditions, the head was held above the water surface, and mice swam as fast as in untethered trials (Figure S3). After two training days, mice reached average escape latencies below $30 \mathrm{~s}$ (Figure $2 \mathrm{~d}$ ). On day 3, they were tested for spatial reference memory (no platform, $60 \mathrm{~s}$ probe trial (PT)) with and without optogenetic silencing of the original encoding ensemble (i.e., cFos+ cells tagged on day 1 ). The experiments were designed to have light ON/OFF probe trials in each mouse, counterbalancing to observe potential light effects (and not trial effects). We quantified the time spent close to the target position (annulus $=$ platform diameter $\times 3.5=35 \mathrm{~cm}$ ) to the time spent in the corresponding area in the opposite quadrant (Figure 2e).

Optogenetic silencing on day 3 had no effect on WM performance (Figure 2f). However, mice had not yet developed full spatial accuracy. Therefore, we trained the mice further until they reached asymptotic escape latencies ( $<20 \mathrm{~s}$ ) and then tested again for potential effects of optogenetic inhibition on day 5 . In the probe trial, all mice showed a spatially accurate search in the target region (Figure 2g). To our surprise, optogenetic silencing of cFos+ cells significantly increased the time spent in the target region (annulus E). We detected no effects of optogenetic inhibition on swim speed or distance moved (Figure 2h, and S3). Apparently, activity in the original encoding ensemble contributed noise rather than useful spatial information and thereby interfered with spatial learning and memory consolidation (Figure $2 \mathrm{~g}$ ). We confirmed that blue light prevented action potentials in iChloC expressing GCs (Figure 2i, and S3) 
bioRxiv preprint doi: https://doi.org/10.1101/2020.08.29.273391; this version posted August 31, 2020. The copyright holder for this preprint (which was not certified by peer review) is the author/funder. All rights reserved. No reuse allowed without permission.

a

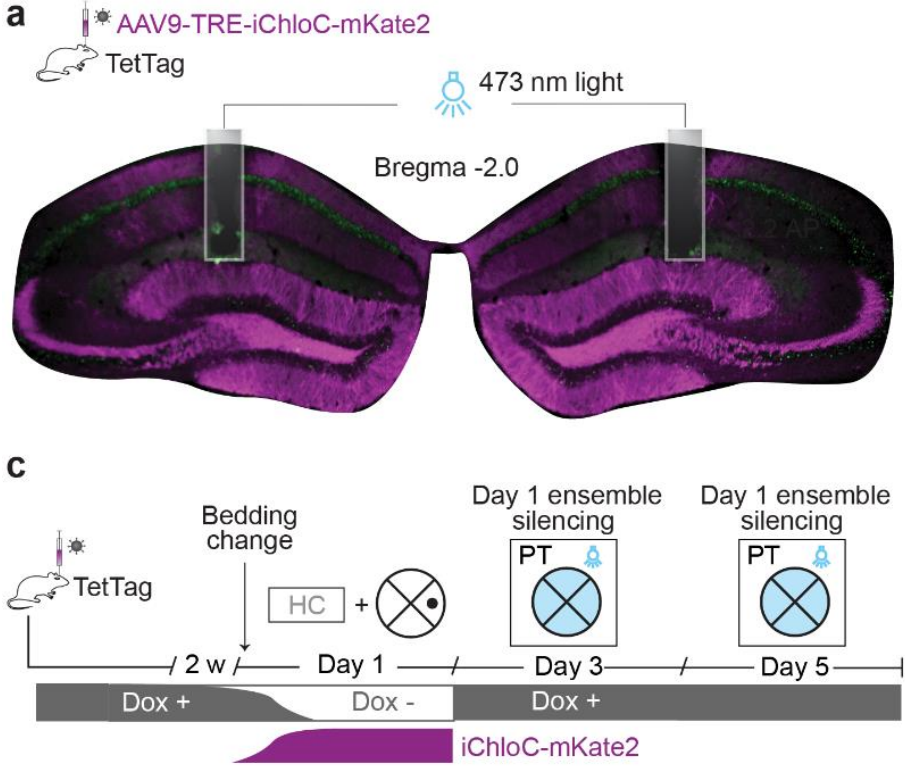

b

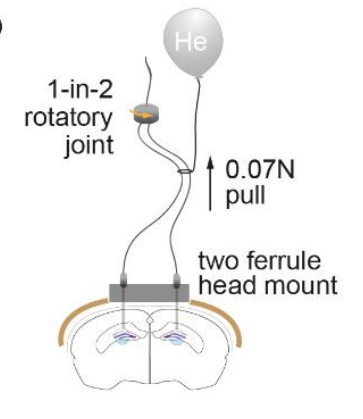

e

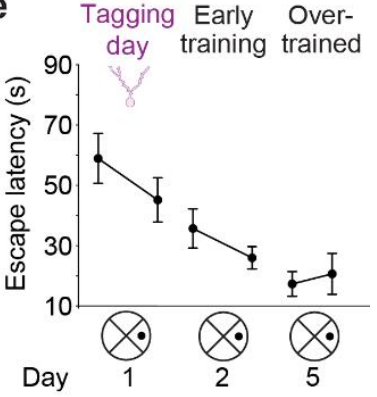

d

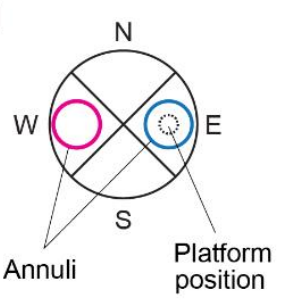

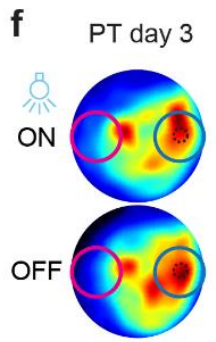

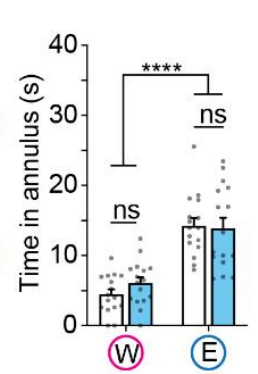

i
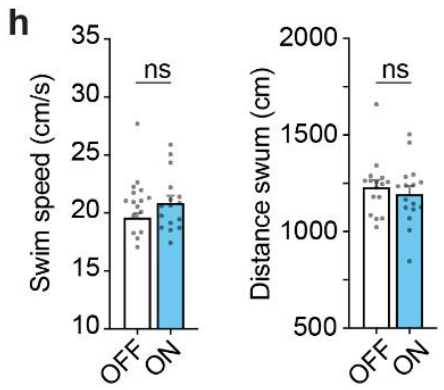
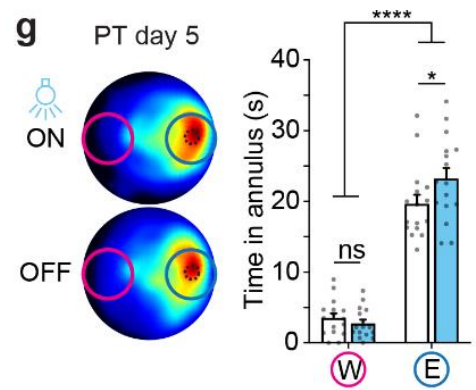

Figure 2. Optogenetic silencing of day 1 cFos-tagged DG ensembles improves spatial memory recall in over trained (day 5) mice but not at early training (day 3) phases of the WM. a, TetTag mice were injected with AAV-TRE-iChloC-mKate2 and a bilateral fiber-optic cannula was implanted above DG. For optogenetic inhibition, $20 \mathrm{~ms}(1 \mathrm{~Hz}), 473 \mathrm{~nm}$ light pulses were used. $\mathbf{b}$, Setup for optogenetic stimulation in the water maze, using calibrated pull-up by helium balloon. c, Experimental design. Mice $(n=16)$ were trained in the WM with a platform in quadrant $\mathrm{E}$, on day 1, off-Dox and put back on-Dox afterward (tagging day). On day 2, mice were trained again with tether, but no optogenetic manipulation was done (early training). Before day 3 training, mice were tethered and were tested twice (probe trial light ON/OFF) for reference spatial memory. Mice were trained untethered 4. On day 5, mice had 2 probe trials with and without light. $d$, Schematic representation virtual divisions of the WM. e, Escape latency at different stages of training. Mice reach asymptotic performance on day 5. f, Counterbalanced (light OFF/ON) probe trials on day 3. Mice show a significant preference for annulus E over $\mathrm{W}\left({ }^{* * *}, \mathrm{p}<0.0001\right.$, effect of annulus position by mixed 2-way-ANOVA) but no interaction between light and time in the annulus $(p=0.47$, matched, 2-way-ANOVA). g, Counterbalanced (light OFF/ON) probe trials on day 5. Mice show a significant preference for annulus $E$ over $\mathrm{W}(* * * *, p<0.0001$, effect of annulus position by mixed 2-way-ANOVA) and light significantly increases the spent time in annulus $E(* p=0.04$, Bonferroni test after mixed 2-way ANOVA: annulus $E$, light OFF vs. ON). $h$, Swim speed and distance swum are not affected by light on day 5. i, Hippocampal slice cultures were used to test the effect of blue light on action potential formation in GCs expressing iChloc. 


\section{Optogenetic silencing during reversal training improves reversal learning}

To test the memory interference hypothesis, we evaluated how silencing of the "old" (day 1) representation affected the acquisition of a new memory. The same cohort $(n=16)$ of AAV-injected TetTag mice (Figure 2 ) was trained on day 6 , but we changed the platform position to the opposite quadrant (W) (reversal training, RT, Figure 3a, c). Mice were separated into two groups with similar performance in the probe trial (light OFF) on day 5 (Figure S3). All mice were tethered; some received light during reversal training (RT-silenced, $n=9$ mice) and some did not (RT-control, $n=7$ mice). Both groups rapidly learned the new escape location, showing a significant decrease in their escape latencies during the training trials (Figure $3 b$ ). We noticed, however, a difference in spatial precision: In the last training trial, silenced mice had developed a clear memory of the new platform location while control mice had not significantly improved compared to the first reversal training trial (Figure $3 d$ ). Thus, silencing neurons associated with the early acquisition (day 1) of the experimental arena and first platform position improved the learning of a new platform position. After the reversal training trials, reference memory was tested in a tethered probe trial without optogenetic silencing. Reversal learning was incomplete, as control animals still had a significant preference for the old platform position (E). In contrast, mice that were trained under inhibition showed a better memory of the new platform position, visiting both old and new platform positions with equal frequency (Figure 6e, f). Analysis of the swimming patterns of individual animals over time revealed that the behavioral difference was most obvious in the first $30 \mathrm{~s}$ of the probe trial: Mice that were trained under inhibition spent significantly more time around the new platform position compared to controls (Figure $3 \mathrm{f}, \mathrm{g}$ ). Thus, optogenetic suppression of the DG ensemble that was active during early training of the first platform position significantly improved reversal learning. Finally, to investigate the effect of optogenetic inhibition on cFos expression, we processed all brains for immuno-enhanced confocal imaging. To estimate the fraction of active neurons in dorsal DG (ensemble size), we normalized the number of cFos+ neurons by the total number of granule cells in each brain section ( 6 sections per animal), estimated from DAPI staining. The size of cFos ensembles was similar on day 1 and day 6 , suggesting the same number of GCs were engaged on both days (Figure 3g). The cellular overlap between ensembles was lower than chance in all mice, suggesting that a different set of GCs was used to represent the events of day 1 and day 6 , despite the environmental familiarity. Mice from the silenced group showed significantly lower overlap than the control group, demonstrating successful optogenetic inhibition of the day 1 ensemble on day 6 (Figure $3 \mathrm{~h}$ ). These results suggest that activity in the originally active ensemble (day 1 cFos+ neurons) interferes with the rapid acquisition of new spatial information several days later. To dissect if the low overlap was due to the temporal distance between tagging days or driven by environmental factors, we decided to evaluate the effects of time, environmental changes, and learning in separate experiments. 
bioRxiv preprint doi: https://doi.org/10.1101/2020.08.29.273391; this version posted August 31, 2020. The copyright holder for this preprint (which was not certified by peer review) is the author/funder. All rights reserved. No reuse allowed without permission.

a

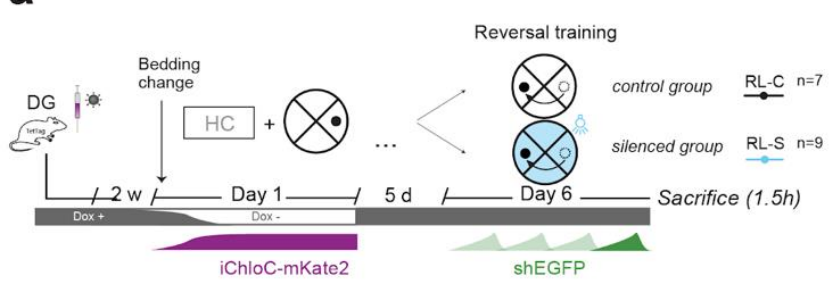

b

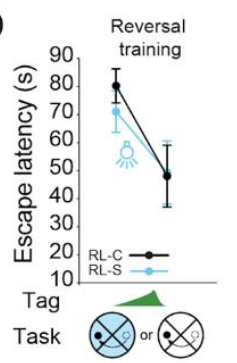

C

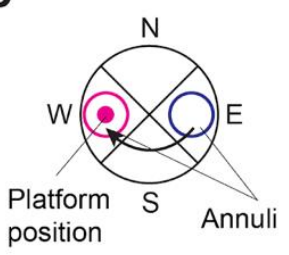

d

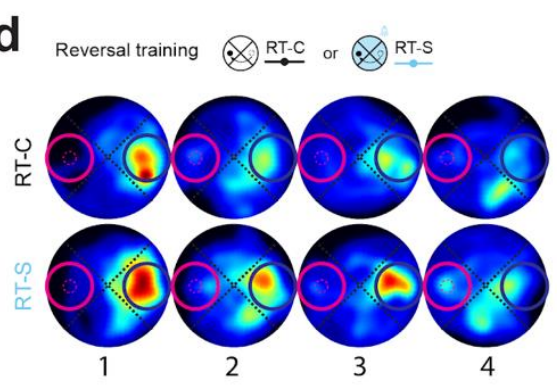

f

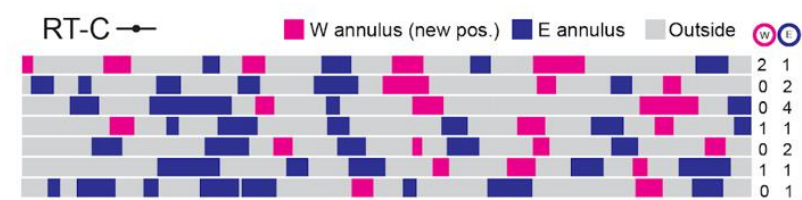

RT-S $\rightarrow$
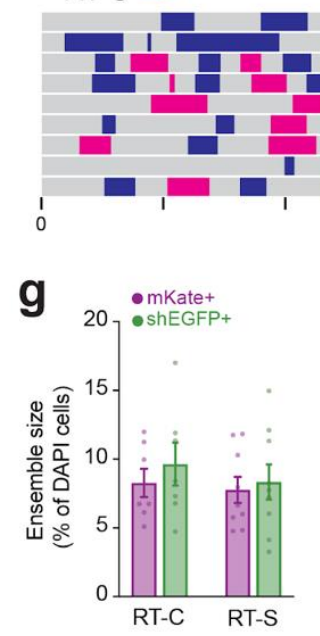

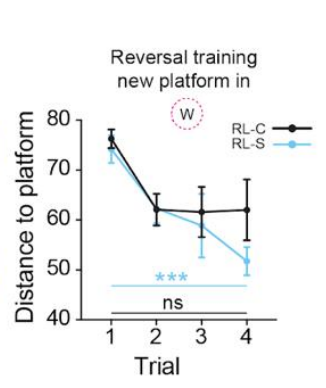

e

PT day $6 \underset{\bigotimes}{\otimes} \cdot$ RLLC.

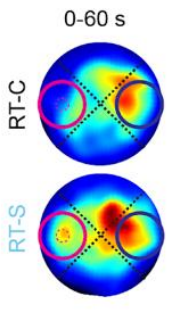

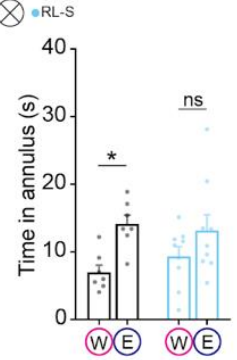

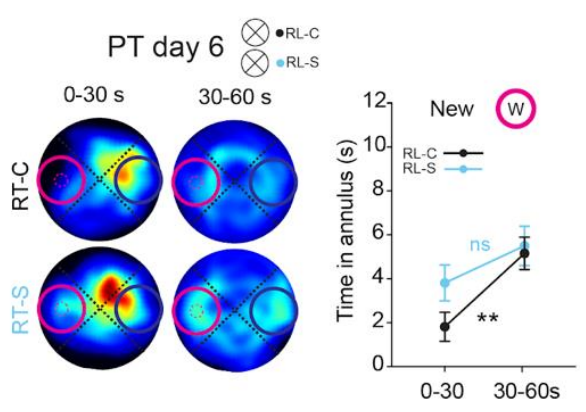

Figure 3. Optogenetic silencing of day 1 cFos-tagged DG ensembles improves reversal training acquisition and recall. a, Experimental design. The same cohort of mice from Figure 2 was separated into two groups with similar performances during the last light OFF PT on day 5. Both groups were tethered and underwent four training trials with the platform in annulus W (reversal training, RT). RT-silenced mice received $20 \mathrm{~ms}, 473 \mathrm{~nm}$ light pulses during the whole length of each learning trial. 1.5h after RT, mice were sacrificed for cFos ensemble immunofluorescence essay. b, Escape latency of each group (only trial 1 and 4 shown). c, Schematic representation of WM tank virtual divisions. $\mathbf{d}$, Shift in search behavior over RT trials on day 6. Heatmaps showing average mouse position for the RT-C (top row) and RT-S (bottom row) groups. Mean minimal distance to the new platform position (W) over the four RT trials. The RT-S group, but not the RT-C group, swim closer to the new platform position during trial four compared to trial one ${ }^{* * *}, \mathrm{p}=0.0004$ trial; Tukey's test after mixed 2-way ANOVA). e, PT 60s, no platform, no light, tethered. Both groups showed similar spatial memory retrieval but mice in the RT$S$ group showed no longer preference for annulus E over W while RT-C does ( ${ }^{*}, p=0.02$ trial; Tukey's test after mixed 2-way ANOVA). $f$, Raster plot of behavior per individual mice during the PT (60 s). Time spent in annuli (W shown in pink, E shown in blue) gray indicates time spent outside annuli. Numbers on the right indicate platform crossings. PT divided in $30 \mathrm{~s}$ time bins shows mice in the RT-S group search in both annuli while mice in the RT-C show a significant preference for annulus E in the first $30 \mathrm{~s}$ of the PT (** $p=0.009$, test $0-30 \mathrm{~s}$ vs. 30-60s, Bonferroni's multiple comparisons after mixed 2-way ANOVA). g, cFos-tagged cells from total of DAPI cells from DG GC layer. 
Both groups show similar ensemble sizes for day 1 (mKate+) and day 6 (shEGFP+). h, overlap/chance was significantly lower in the RT-S group $(*, p=0.03$, unpaired $t$-test). $i$, Venn diagram shows ensemble overlap is minimal in both groups.

\section{In a constant environment, GC ensemble selection is a function of time.}

In vivo recordings of GCs indicate that the ones that encode spatial information show low remapping across time in the same environment and high remapping in different environments (Danielson et al., 2017; GoodSmith et al., 2017; Rennó-Costa et al., 2010; van Dijk \& Fenton, 2018). Our expectation was, therefore, that cFos+ neuronal ensembles would remain stable over time as long as the setting is not changed. To test this hypothesis, we used TetTag mice injected with AAV9-TRE-iChloC-mKate2 which were kept in their home cage throughout the experiment. We sacrificed the animals at 3 different time points after the end of the off-Dox period: Immediately $(\Delta 0 h, n=3)$, after $6 h(\Delta 6 h, n=$ $3)$, and after $24 \mathrm{~h}(\Delta 24 \mathrm{~h} n=4)$. In the $\Delta 0 \mathrm{~h}$ group, $24 \%$ of tagged GCs were double-positive. The smaller mKate+ ensemble size in the $\Delta 0 \mathrm{~h}$ and $\Delta 6 \mathrm{~h}$ groups compared to the $\Delta 24 \mathrm{~h}$ group, in contrast to the consistent shEGFP+ ensemble size in all groups, confirmed that the TRE-driven construct took at least $24 \mathrm{~h}$ to reach full expression (Figure $4 \mathrm{a}, \mathrm{c}$ and Figure $1 \mathrm{~d}, \mathrm{e}$ ). The number of double-positive cells was similar in the $\Delta 6 \mathrm{~h}$ and $\Delta 0 \mathrm{~h}$ groups, indicating that active neuronal ensembles are stable over short ( $<6 \mathrm{~h}$ ) periods. In contrast, the $\Delta 24 \mathrm{~h}$ group showed a surprisingly low number of doublepositive cells (Figure 4c). To compensate for the different sizes of the first encoding ensemble at the different time points, we compared the actual number of double-positive cells to the expected number if the second set of neurons had been randomly selected (chance level, Figure $4 d$ ). The degree of overlap was much higher than expected by chance for the $0 \mathrm{~h}$ and $6 \mathrm{~h}$ groups, suggesting that the same neuronal ensemble was active at two time points within a short time window. After 24 $h$, the overlap between the ensembles had fallen slightly below chance level, suggesting that even in a constant environment (i.e., the home cage), a new set of GCs is used for memory encoding on consecutive days (Figure 4d). As even after 5 days of water maze training, the overlap was not further reduced (Figure $3 \mathrm{~h}$ ), we suggest that ensemble reactivation may be a sigmoidal function of time, dropping to chance levels after $\sim 12 \mathrm{~h}$ (Figure $4 \mathrm{e}$ ).

In addition to counting shEGFP+ neurons, we analyzed the intensity of green fluorescence in these neurons to estimate the degree of activation. We found the highest expression levels in doublepositive cells sacrificed right at the end of the off-Dox labeling window ( $\Delta \mathrm{Oh}$, Figure $4 \mathrm{f}$ ). This is not surprising: As the labeling windows overlapped for several hours, selecting mKate-positive GCs biased the sample towards highly active neurons with high cFos levels. After $6 \mathrm{~h}$ on-Dox, shEGFP expression levels in double-positive GCs was already reduced, and after $24 \mathrm{~h}$ on-Dox, the difference between shEGFP expression in double-positive GCs and in GCs expressing only shEGFP had completely disappeared. This analysis of expression levels confirmed our conclusion from the overlap analysis, that individual GCs do not maintain high cFos levels for more than a few hours and are rarely reactivated, causing the global pattern of cFos expression in DG to change over time.

In GCs expressing only shEGFP (the large majority of shEGFP+ cells), expression levels were independent of the time point of sacrifice, as expected (Figure 4f). Together with the constant size of 
the shEGFP ensemble, this indicates that Dox withdrawal and bedding change had little or no effect on the overall activity in DG.

a
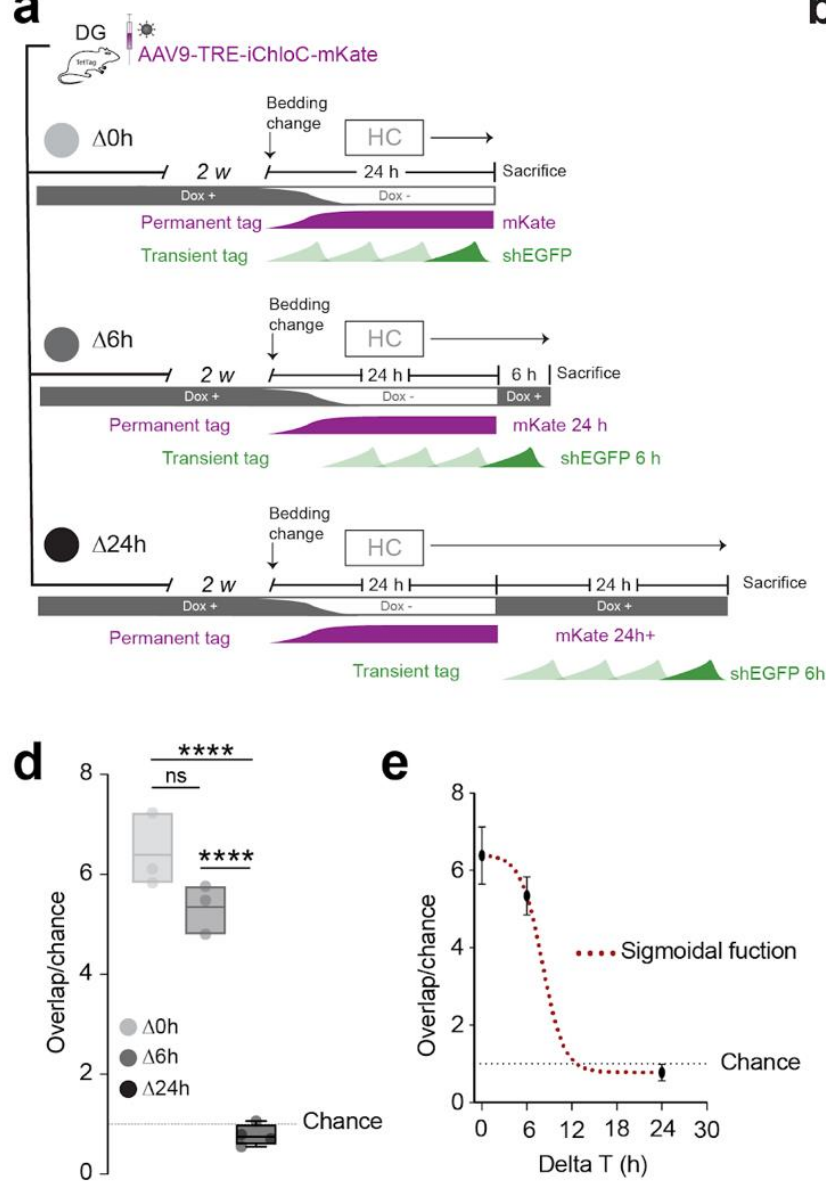

e

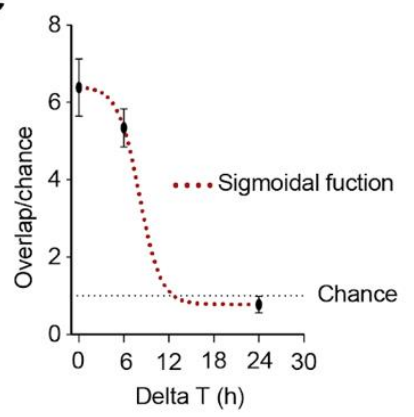

b
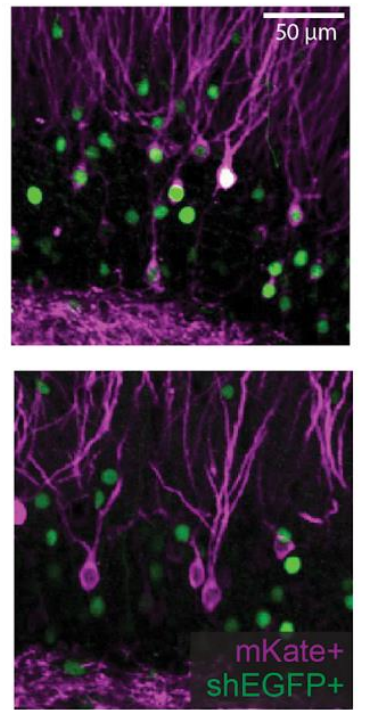

C

shEGFP+ mKate $^{+}$

ODouble+(Overlap)

$\Lambda \mathrm{Oh}$<smiles>Cc1ccc([AsH2])cc1</smiles>

$24 \%$

$\Delta 6 \mathrm{~h}$

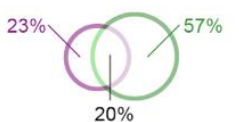

$2^{124 h}{ }^{46 \%} C_{3 \%}^{49 \%}$

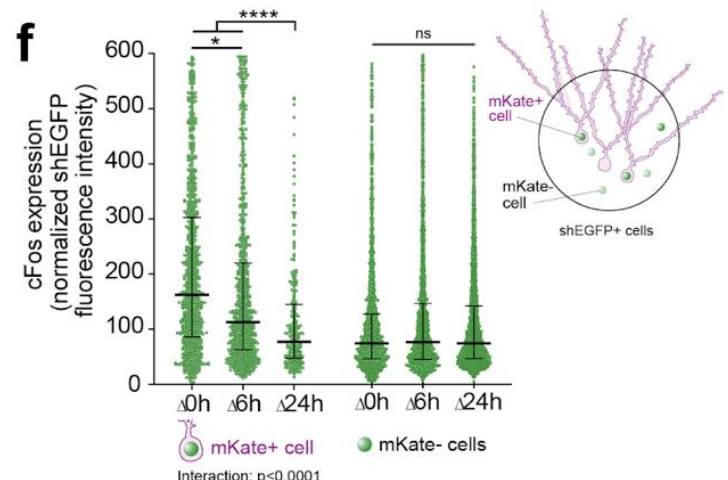

Figure 4. In the familiar home cage (HC), cFos+ GC ensembles strongly segregate over time. a, Experimental design: TetTag mice were injected with AAV-TRE-iChloC-mKate. Dox was removed (off) for $24 \mathrm{~h}$ and mice were sacrificed immediately after $(\Delta 0 \mathrm{~h}$ group, $\mathrm{n}=3$ ). Dox was given back (on) and mice were either sacrificed 6 ( $\Delta 6 \mathrm{~h}$ group, $n=3$ ) or $24 \mathrm{~h}$ after ( $\Delta 24 \mathrm{~h}$ group, $n=4)$. $b$, Representative confocal images of cFos-tagged GCs. cFos-tagged cells during the off-Dox period express mKate (pseudo-colored in magenta). Transient tagged cFos GCs from hours prior to sacrifice express shEGFP (green). c, Venn diagram of tagged cells show ensemble overlap. d, Overlap/chance is similar in the $\Delta 0 \mathrm{~h}$ and $\Delta 6 \mathrm{~h}$ groups (ns, $\mathrm{p}=0.11$, unpaired t-test). Both the $\Delta 0 \mathrm{~h}$ and $\Delta 6 \mathrm{~h}$ groups have significantly higher overlap/chance than the $\Delta 24 \mathrm{~h}$ group ( ${ }^{* * *}, \mathrm{p}<0.0001$, unpaired t-test). e, Overlap/chance follows a sigmoidal function over time. $\mathrm{f}$, Double-labeled GCs $(\mathrm{mKate}+/ \mathrm{shEGFP+})$ expressed shEGFP significantly stronger immediately after the bedding change $(\Delta 0 \mathrm{~h})$ compared to $6 \mathrm{~h}$ later $(*, \mathrm{p}=$ $0.01)$, or $24 \mathrm{~h}$ later $(* * *, \mathrm{p}=0.01)$. In GCs expressing only shEGFP, not mKate, expression levels were identical at all time points (twoway-ANOVA, Tukey).

\section{cFos ensembles get minimally reactivated during spatial learning}

The unexpectedly low overlap of the cFos+ ensembles in a constant environment could have been caused by the fact that hippocampal circuitry is barely engaged when mice are in the home cage. Thus, we next tested whether the amount of overlap increases when animals explore a new environment or develop a spatial navigation strategy. Given the strong effect that time has on the 
reactivation of cFos ensembles, we kept a consistent $24 \mathrm{~h}$ interval between cFos-tagging events. AAV-injected mice were taken off-Dox and trained in the WM with a platform in the E quadrant. After training, mice were fed again with Dox and, $24 \mathrm{~h}$ later, they either were trained in the WM with the same platform position (early training, ET) or allowed to explore an unfamiliar open field (novel environment, NE, Fig 5a). To maximize novelty, the NE group was tested in a different experimental room and handled by a different researcher. On day 1 , mice in both groups showed similar escape latencies in the water maze. On day 2, only mice from the ET group were further trained in the WM, showing a further significant decrease in escape latency (trial 1 vs trail 8, Figure 5b). Reference memory was tested on both days in probe trials without the platform (Figure 5c). On day 1, mice did not show any significant preference for the platform position (annulus E) when compared to the opposite side of the maze (annulus W). At the end of training day 2, the ET group had learned the task as indicated by a significant preference for the target annulus (Figure $5 c$ ). Three hours after the last trial, mice were sacrificed for cFos ensemble analysis. As in the previously described experiments, we counted tagged cells and compared the number of double-positive neurons to the expected overlap for randomly selected neurons. cFos+ ensemble size was similar in both groups for both days (Figure $5 d$, and S4). 
a

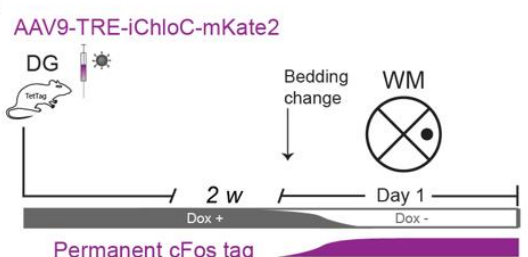

Permanent cFos tag

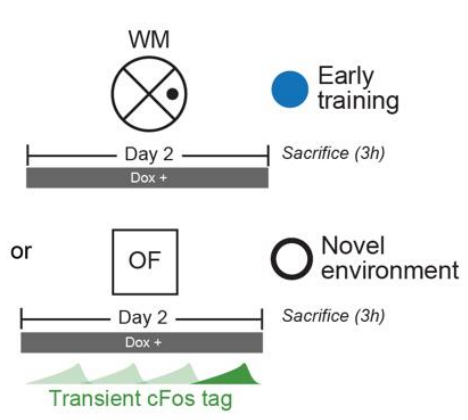

b

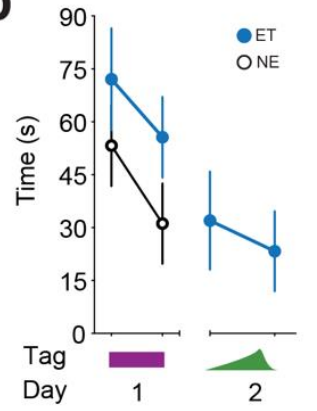

C

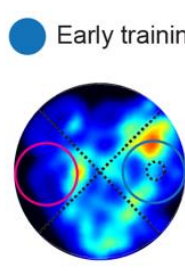

Tag
1

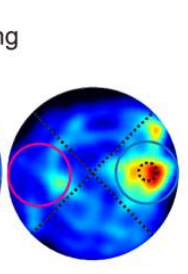

2

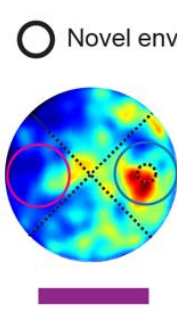

1

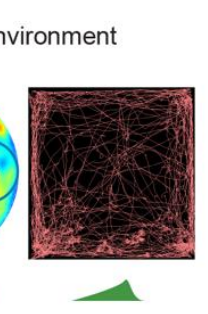

2
Annulus

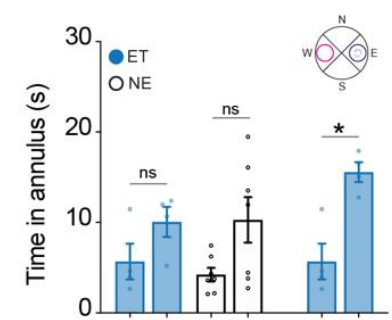

Day

1
2 e

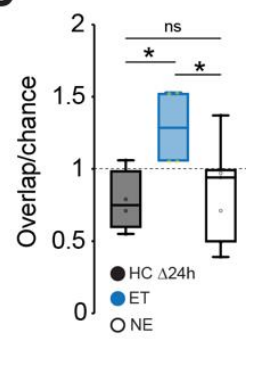

d

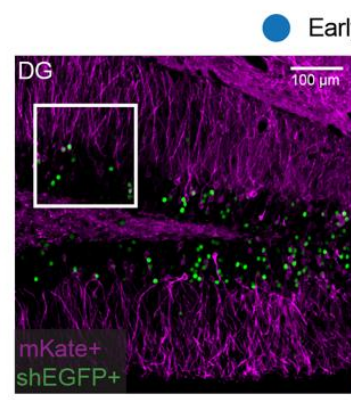

Early training

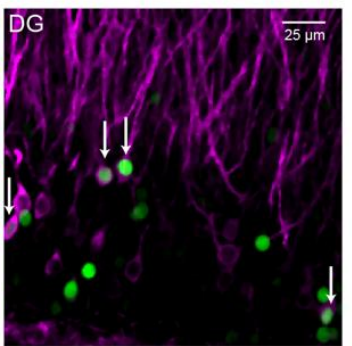

Novel environment
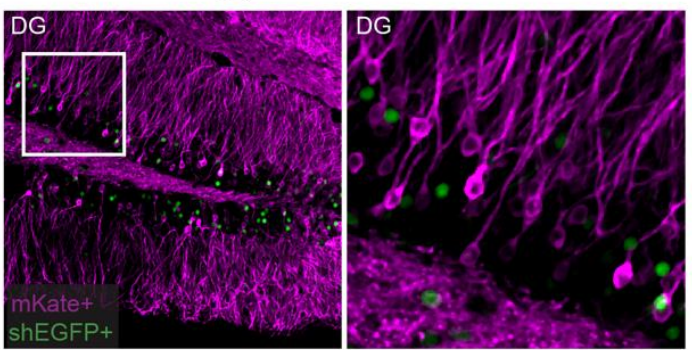

Figure 5. cFos+ GC ensembles get partially reactivated during early training phases of WM but segregate when mice visit an unfamiliar environment. a, Experimental design. TetTag mice were injected with AVV-TRE-iChloC-mKate in DG and trained while Dox (-) in the WM (platform in E). Mice were then put back on Dox (+) and 24h later, they were either trained again in the WM (early training, ET) or visited an open field arena in a different room for 20 mins (novel environment, NE). Mice were sacrificed $3 \mathrm{~h}$ after the last trial for cFos-tagged GCs immunofluorescence essay. b, Escape latencies from the first and last trial of day 1 , show similar learning in both groups. Mice in the ET group significantly reduced their escape latency from day 1 to day $2\left(^{*}, p<0.05\right.$, repeated measures one-way-ANOVA test). c, Reference memory PT, no platform. Heatmaps from each day per group. Representative OF track of one mouse from the NE group on day 2. $d$, Quantification of time in each annuli during PT. On day 1, mice show similar spatial reference memory performance in both groups but no significant preference for annulus E. On day 2, mice from the ET group show a significant preference for annulus $E(*$, $p<0.05, \mathrm{RM}$-twoway-ANOVA). e, Representative confocal images of DG GC layer. ET, top; NE bottom. Arrows indicate GCs with cFos on both days. f, Ensembles size. Both groups show similar ensembles size for both days. g, Overlap/chance is significantly higher in the ET group than the NE or HC $\Delta 24$ h group from Figure $4 .{ }^{*}, p<0.05$, t test)

Overlap/chance was significantly higher in the ET group than both the NE and $24 \mathrm{~h} \mathrm{HC} \mathrm{group,}$ suggesting that mice do reactivate yesterday's GCs during multi-day spatial learning (Figure $5 \mathrm{~d}$, e). Exposure to a novel environment without any link to the previously trained water maze task did not drive ensemble reactivation in DG. The number of cFos ${ }^{+}$neurons labeled on day 2 (shEGFP) did not differ between the three groups. However, within the set of shEGFP-expressing neurons, expression levels were significantly higher in the ET group compared to the NE and home cage groups (Figure S4). These results suggest that spatial navigation, a hippocampus-dependent task, activates GCs 
more strongly than the exploration of a new environment, even though the total number of cFos+ neurons was constant under all conditions. Although significantly higher than the NE and $\Delta 24 \mathrm{~h}$ groups, reactivation levels in the ET group were still low compared to the $\Delta 0 \mathrm{~h}$ or $\Delta 6 \mathrm{~h}$ home cage groups.

\section{Well-trained animals facing a novel challenge}

Comparing day 1 and day 2 of WM training, we found little overlap between cFos ensembles, but the behavioral performance on day 1 was also very poor. What about two consecutive training days in mice that are already experts at finding the hidden platform? We used AAV-injected mice that were kept on-Dox during the first 4 training days, were trained off-Dox on day 5, and put back on Dox immediately after training. On day 6, mice were either trained again (overtraining, OT) or trained with the platform in the opposite quadrant (W) to test the spatial interference (reversal training, RT, Figure 6a). Both groups showed excellent performance on day 5 , the first tagging day. Mice from both groups did not further improve during this day, indicating asymptotic learning (escape latency < $15 \mathrm{~s})$.

On day 6, mice of the OT group maintained their performance, and RT mice improved their performance during the 4 reversal training trials (Figure 6b). Spatial reference memory was tested in a probe trial at the end of each training day. On day 5 , mice in both groups showed spatially precise search behavior, swimming in the proximity of the platform position (annulus E). On day 6, the OT group preferentially searched at annulus E while the RT group searched at both the old (annulus E) and new (annulus W) platform positions (Figure 6c, e). The restricted spatial search was evident in the heat maps (averaged over all animals) and could also be seen in the behavior of each individual mouse during the probe trials of day 5 and day 6 (Figure $6 \mathrm{~d}$ ). Mice were sacrificed $3 \mathrm{~h}$ after the last trial and brains were processed for cFos mapping. Consistent with previous experiments, we observed similar ensemble sizes from both days in both groups (Figure S4). Contrary to our expectation, ensemble overlap in the OT group was very low, comparable to the $\Delta 24 \mathrm{~h} \mathrm{HC}$ group. In contrast, the RT group showed a significantly higher overlap than the $\Delta 24 \mathrm{~h} H \mathrm{HC}$ group. The spatial search consistency between the two tagging days was high in the OT group, but ensemble reactivation was low. In contrast, spatial search consistency in the RL was low, but the ensemble reactivation was high. These results indicate that the pattern of cFos+ cells in DG contains little information about the spatial position of the animal. 
bioRxiv preprint doi: https://doi.org/10.1101/2020.08.29.273391; this version posted August 31, 2020. The copyright holder for this preprint (which was not certified by peer review) is the author/funder. All rights reserved. No reuse allowed without permission.

a

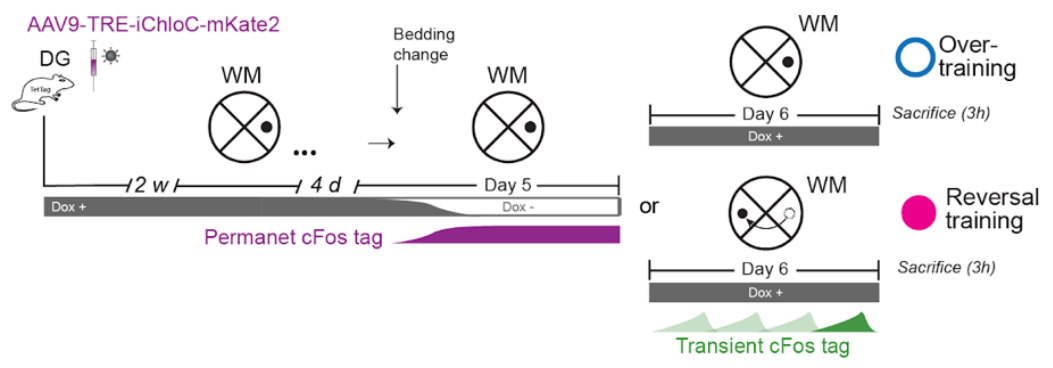

b

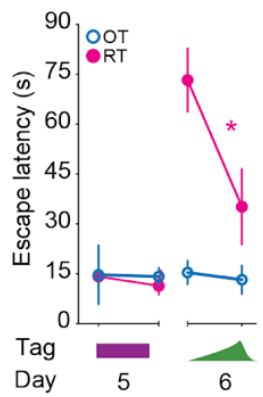

C

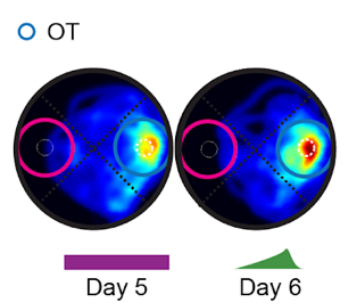

- RT

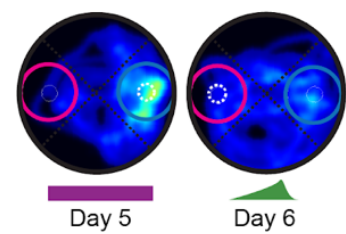

d

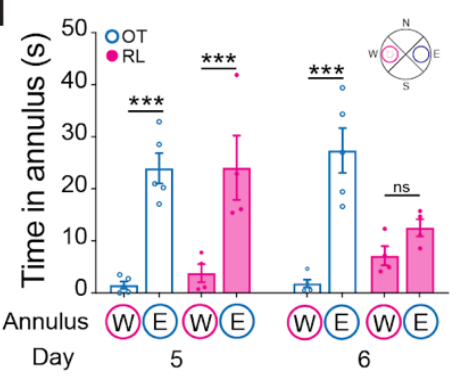

Оот

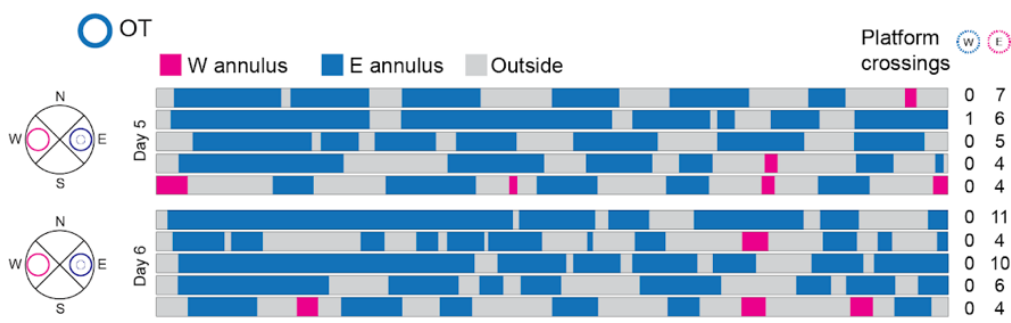

RT

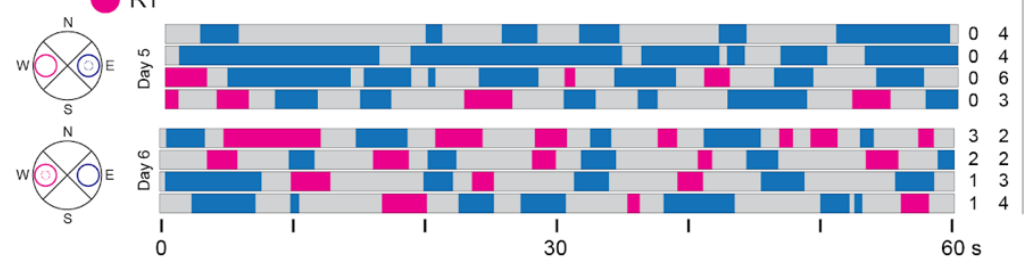

f

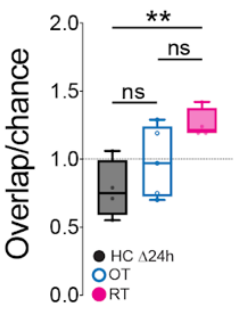

Figure 6. cFos-tagged GCs poorly reflect mouse location. a, Experimental design. TetTag mice were injected bilaterally in DG with AAVTRE-iChloC-mKate. Mice were trained in the WM with the platform in E for 4 consecutive days with on-Dox. On day 5 , mice were trained with the same platform position off-Dox. Mice were put back on-Dox and on day 6 they were either trained again with the platform in $E$ (overtrained, OT) or with the platform in W (reversal training, RT). Mice were sacrificed $3 \mathrm{~h}$ after the last trial for cFos-tagged GCs immunofluorescence essay. b, Escape latencies show similar learning in both groups on day 5 . The RT group significantly reduced the escape latency during day $6\left(^{*}, p<0.05\right.$, repeated measures one-way-ANOVA test. c, Heatmaps represent the PTs on day 5 and 6 for the OT and RT groups d, Raster plot of time spent at the W annulus (pink), E annulus (blue), and outside the annuli (gray) for each mouse of the OT and RT groups during the PT on day 5 and 6 . Numbers on the right indicate platform crossings. e, Time in each annulus during the PTs. On day 5, mice show similar performance in both groups with a highly significant preference for annulus E. On day 6 , the RT group shows no significant preference for any annulus, while the OT group keep their preference for annulus $E\left({ }^{*}, p<0.05, t\right.$ test). $f$, Overlap/chance is significantly higher in the RT than the $\Delta 24 \mathrm{HC}$ group (**, p=0.006, unpaired, two-tailed t-test). 


\section{Discussion}

We used activity-dependent expression of a light-gated chloride channel to evaluate how cFos+ ensembles in the dentate gyrus contribute to spatial memory. Optogenetic inhibition of ensembles that were active during early training (day 1 ) improved memory recall in overtrained animals (day 5) and improved memory acquisition of a new platform location (reversal training, day 6). Even in animals very familiar with the WM, cFos+ ensembles in DG strongly segregated over time, indicating that the pattern of cFos+ expression does not represent the animal's location in space. However, when spatial learning took place, either at early training stages or during reversal training, cFos+ ensembles were partially reactivated. The slow dynamics of cFos+ ensembles in DG may reflect its role in time stamping of memories.

\section{How does DG contribute to spatial memory?}

Lesion studies and pharmacological experiments first established that the DG is important for the encoding of spatial memories, but provided no information about activity patterns during navigation (Lassalle et al., 2000; Lee \& Kesner, 2004). More recently, optogenetic tools were employed to investigate DG function by hyperpolarize hilar interneurons (Andrews-Zwilling et al., 2012), adultborn granule cells (Gu et al., 2012) or projections from the entorhinal cortex (Qin et al., 2018) during water maze training. Optogenetic hyperpolarization of GABAergic interneurons removes the lateral inhibition of GCs, leading to a relatively dense activity of GCs and compromised spatial memory recall (Andrews-Zwilling et al., 2012). Direct hyperpolarization of adult-born GCs or block of the inputs from layer II of medial entorhinal cortex by axonal hyperpolarization also impaired spatial memory recall (Gu et al., 2012) (Qin et al., 2018).

These still fairly global interventions suggest that both too dense and too weak GC activity degrades behavioral performance. In addition to water maze experiments, active place avoidance has been used to study the importance of DG for spatial learning (Kheirbek et al., 2013). In conflict trials, animals with inhibited dorsal DG often entered the shock zone and spent less time in the new 'safe' quadrant, indicating impaired discrimination. In contrast, we found that optogenetic inhibition during reversal training improved reversal learning (Figure 3). There are, however, major differences in the experimental design: While Kheirbeck et al. used a global expression strategy (Pomc-cre) to drive eNpHR3.0 in all GCs, we expressed the optogenetic inhibitor only in the small subset of GCs $(\sim 10 \%)$ that were active during the first WM-encoding episode. Therefore, Kheirbeck et al. shut down all activity in a large part of the DG while our manipulation left the processing intact in $>90 \%$ of GCs, resulting in opposite effects on spatial learning.

\section{The effect of optogenetic silencing depends on the behavioral paradigm}

Specific inhibition of previously active neurons in DG has already been used in the context of fear conditioning, a model for posttraumatic stress disorder (PTSD). Optogenetic silencing of arc-tagged ensembles in DG prevented the associated behavioral fear response to a particular encoding context 
(Denny et al., 2014; Lacagnina et al., 2019). In contrast, when we silenced the original encoding ensemble in the water maze, we observed improvement in the recall of the first platform position (Figure 2g) and better acquisition of a new spatial location (Fig $3 \mathrm{~d}-\mathrm{f}$ ). Importantly, in contextual fear conditioning experiments, arc-expressing GC populations during induction and recall of fear were overlapping (3-6 fold above chance), while in our spatial memory experiments, the overlap was below chance levels. Given the low reactivation between cFos+ ensembles, it is perhaps not surprising that optogenetic silencing of cFos+ neurons from day 1 (first WM-encoding episode) did not suppress spatial memory recall on day 6 .

\section{Which factors control cFos+ ensemble reactivation in DG?}

Here we report near-constant numbers of cFos+ neurons (ensemble size) under all tested conditions, but consistently higher c-Fos expression (anti-shEGFP fluorescence) in water maze groups compared to open field and home cage controls (Figure S4). This suggests spatial navigation does not increase the number of active GCs, but leads to higher activity levels within the active subset. When comparing the impact of environment, task, and elapsed time on the ensemble reactivation, it became clear that time is the dominant factor driving cFos ensembles apart (Figure 7). However, time is not the only extrinsic factor controlling GC reactivation. Comparing ensemble overlap in all experimental groups with a time interval of $24 \mathrm{~h}$ between the two ensembles ( $\Delta 24 \mathrm{~h}$ ), we noted that relatively high reactivation occurred in situations where the learning curve was very steep (ET: $14 \%$, RT: $12 \%$ ). Low reactivation (below chance) was found in familiar environments (HC: $10 \%, \mathrm{OT}$ : $9 \%$ ) but also in entirely novel environments (NE: 8\%), generally in situations where a recall of yesterday's events would have had no benefit for the animal (Figure 7). The strong influence of time over cFos+ ensemble selection supports the hypothesis that DG processes a familiar environment as novel when experienced temporally separated to aid detecting new features of a known environment for pattern separationDeng et al., 2013; Rangel \& Eichenbaum, 2013). 


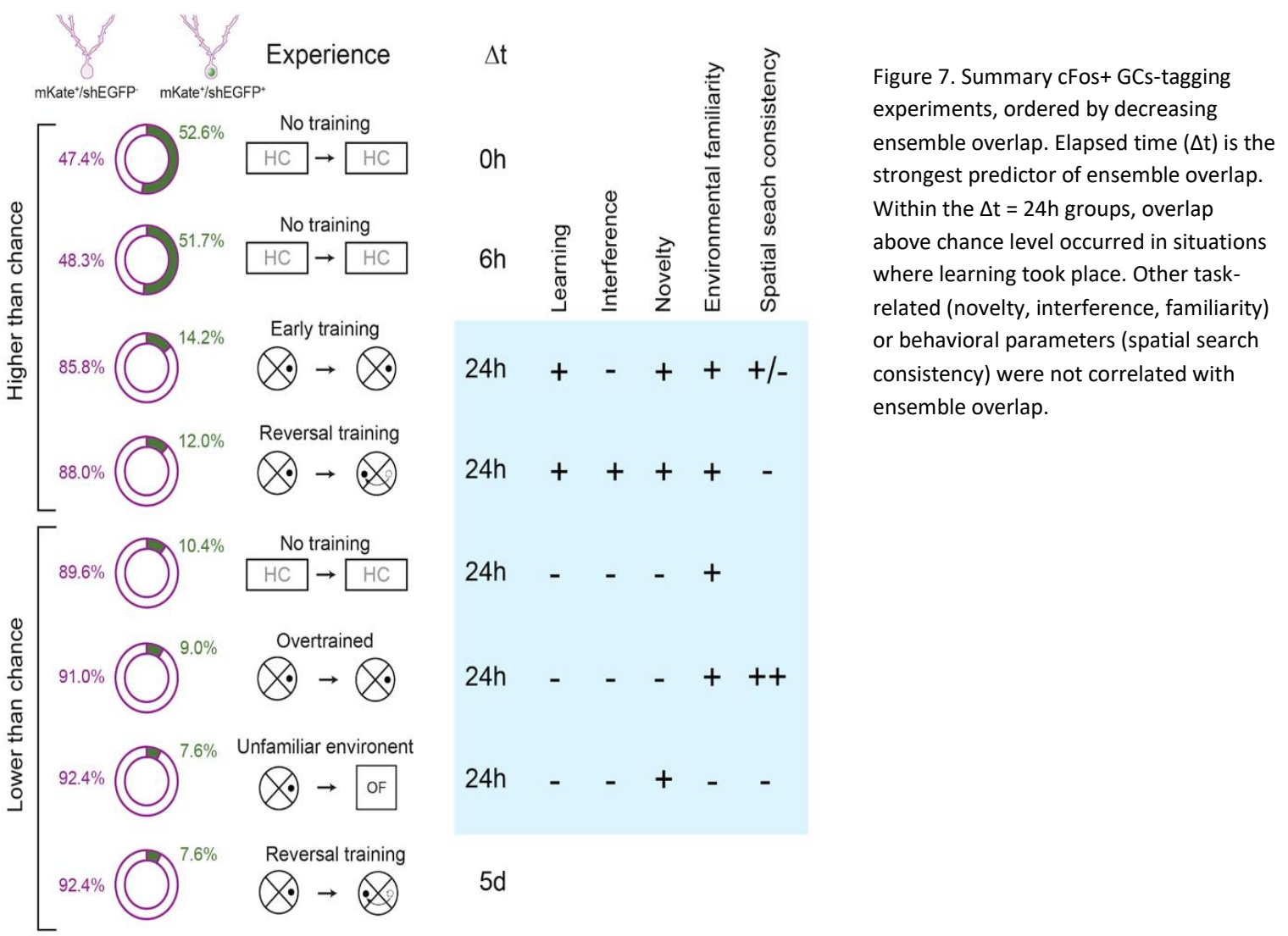

Strikingly, in our results, environmental familiarity alone did not produce ensemble overlap above chance in over trained animals (OT group) even when cFos+ maps were generated only one day apart (Figure 6). One hypothesis is that cFos + cells on day 5, when the animals were already experts in navigating the maze and did not improve their performance any more (asymptotic performance), did not carry information required for solving the task. During reversal training on day 6 , mice partially reactivated the day 5 ensemble. A plausible interpretation is that mice facing an unexpected situation try to recall past experiences to develop a new strategy. The low overlap in the OT group was quite surprising because it has been reported that GCs with strong place preference have highly stable place fields in familiar environments (GoodSmith et al., 2017; Hainmueller \& Bartos, 2018; Leutgeb et al., 2007). Our results suggest that the pattern of cFos activity in DG does not indicate the position of the animal or the goal, at least not in the form of labeled line code. It could be argued that cFos+ neurons from early training are still active in the second labeling window, but not enough to drive cFos (Mahringer et al., 2019). However, when we suppressed activity in the ensemble expressing cFos during initial training via optogenetic inhibition (Figure $2 \mathrm{~g}$ ), animals were perfectly capable of swimming to the correct platform position. At day 5 of WM training, we even saw slightly enhanced performance compared to non-inhibited trials. This would not be expected if the cFos+ cells from day 1 still encoded specific locations in the water maze on day 3 and 5. 


\section{cFos is part of a mechanism that selects neurons for memory storage}

When we evaluated shEGFP expression levels across home cage groups (Figure 2), we found that double-positive neurons (shEGFP+ / mKate+) in the $\Delta 0 \mathrm{~h}$ group had the highest fluorescence intensity, even higher than the WM groups (Figure. 4f, and S4). The effect was less pronounced in the $\Delta 6 \mathrm{~h}$ group and even less in the $\Delta 24 \mathrm{~h}$ group. In contrast, shEGFP expression levels in non-reactivated neurons (mKate-) were similar in all home cage groups (Figure. 4f). This observation suggests that DG neurons that were active early in the off-dox period (accumulating mKate) also had the highest activity levels towards the end (resulting in very high shEGFP expression), but not 24 hours later. It is very likely that the reported hyperexcitability of cFos-expressing neurons, caused by $\mathrm{K}^{+}$channel internalization (Pignatelli et al., 2019), is responsible for the correlation (at 0 and $6 \mathrm{~h}$ ) between the fast/transient (shEGFP) and slow/permanent (mKate) cFos reporter. It is less clear how the period of elevated activity is terminated. High expression of another immediate early gene, homer1a, has been shown to trigger cell-wide synaptic downscaling (Diering et al., 2017). As it takes several hours for this homeostatic mechanism to take effect, it is a good candidate to explain the slow migration of DG ensemble activity over time. Importantly, this type of cell- autonomous oscillation would provide a clock that reduces synaptic drive specifically for the most active subset of neurons but has little effect on the $90 \%$ of GCs that are cFos-negative. A cell-specific negative feedback loop between immediate-early gene (IEG) activation and excitatory drive could also explain why we saw overlap below chance levels in several experimental groups, which can only be explained if information about the identity of once-active (cFos+) cells is still present in the system. Time-dependent switching to a new set of active GCs might indeed be crucial to distinguish similar experiences that occurred separated in time (Josselyn \& Frankland, 2018; Park et al., 2016). In hippocampus (Cai et al., 2016) and amygdala (Rashid et al., 2016), IEG-expressing neuronal ensembles fail to encode two different events as different experiences when they occur close in time $(<6 \mathrm{~h})$. Spatial or auditory cues were only discriminated when associated with experiences that occurred distant in time. These observations and our data suggest that the temporal shift in ensemble selection occurs even when the spatial context remains the same. Thus, cFos is not only an indicator of intense neuronal activity but also reflects a clock signal (Frank, 2016; McCauley et al., 2019) that is crucial for LTP, perhaps selecting a specific subset of neurons for memory storage. Our results support the concept that cFos+ neurons in DG encode an episode, but not a spatial position (Hainmueller \& Bartos, 2020). The disconnect between cFos expression and place cell activity has also been reported in a recent study of CA1 pyramidal cells (Poll et al., 2020; Tanaka et al., 2018) and is therefore not a specific property of DG granule cells. 


\section{References}

Andrews-Zwilling, Y., Gillespie, A. K., Kravitz, A. V., Nelson, A. B., Devidze, N., Lo, I., Yoon, S. Y., BienLy, N., Ring, K., Zwilling, D., Potter, G. B., Rubenstein, J. L. R., Kreitzer, A. C., \& Huang, Y. (2012). Hilar GABAergic interneuron activity controls spatial learning and memory retrieval. PloS One, 7(7), e40555.

Aronov, D., Nevers, R., \& Tank, D. W. (2017). Mapping of a non-spatial dimension by the hippocampal-entorhinal circuit. Nature, 543(7647), 719-722.

Bernstein, H. L., Lu, Y.-L., Botterill, J. J., \& Scharfman, H. E. (2019). Novelty and Novel Objects Increase c-Fos Immunoreactivity in Mossy Cells in the Mouse Dentate Gyrus. Neural Plasticity, 2019, 1815371.

Bird, C. M., \& Burgess, N. (2008). The hippocampus and memory: insights from spatial processing. Nature Reviews. Neuroscience, 9(3), 182-194.

Cai, D. J., Aharoni, D., Shuman, T., Shobe, J., Biane, J., Song, W., Wei, B., Veshkini, M., La-Vu, M., Lou, J., Flores, S. E., Kim, I., Sano, Y., Zhou, M., Baumgaertel, K., Lavi, A., Kamata, M., Tuszynski, M., Mayford, M., ... Silva, A. J. (2016). A shared neural ensemble links distinct contextual memories encoded close in time. Nature, 534(7605), 115-118.

Chavlis, S., \& Poirazi, P. (2017). Pattern separation in the hippocampus through the eyes of computational modeling. Synapse, 71(6). https://doi.org/10.1002/syn.21972

Danielson, N. B., Kaifosh, P., Zaremba, J. D., Lovett-Barron, M., Tsai, J., Denny, C. A., Balough, E. M., Goldberg, A. R., Drew, L. J., Hen, R., Losonczy, A., \& Kheirbek, M. A. (2016). Distinct Contribution of Adult-Born Hippocampal Granule Cells to Context Encoding. Neuron, 90(1), 101-112.

Danielson, N. B., Turi, G. F., Ladow, M., Chavlis, S., Petrantonakis, P. C., Poirazi, P., \& Losonczy, A. (2017). In Vivo Imaging of Dentate Gyrus Mossy Cells in Behaving Mice. Neuron, 93(3), 552559.e4.

Deng, W., Mayford, M., \& Gage, F. H. (2013). Selection of distinct populations of dentate granule cells in response to inputs as a mechanism for pattern separation in mice. elife, 2, e00312.

Denny, C. A., Kheirbek, M. A., Alba, E. L., Tanaka, K. F., Brachman, R. A., Laughman, K. B., Tomm, N. K., Turi, G. F., Losonczy, A., \& Hen, R. (2014). Hippocampal memory traces are differentially modulated by experience, time, and adult neurogenesis. Neuron, 83(1), 189-201.

Diering, G. H., Nirujogi, R. S., Roth, R. H., Worley, P. F., Pandey, A., \& Huganir, R. L. (2017). Homer1a drives homeostatic scaling-down of excitatory synapses during sleep. Science, 355(6324), 511515.

Eichenbaum, H. (2014). Time cells in the hippocampus: a new dimension for mapping memories. Nature Reviews. Neuroscience, 15(11), 732-744.

Frank, M. G. (2016). Circadian Regulation of Synaptic Plasticity. Biology, 5(3). https://doi.org/10.3390/biology5030031

Gee, C. E., Ohmert, I., Wiegert, J. S., \& Oertner, T. G. (2017). Preparation of Slice Cultures from Rodent Hippocampus. Cold Spring Harbor Protocols, 2017(2). https://doi.org/10.1101/pdb.prot094888

GoodSmith, D., Chen, X., Wang, C., Kim, S. H., Song, H., Burgalossi, A., Christian, K. M., \& Knierim, J. J. (2017). Spatial Representations of Granule Cells and Mossy Cells of the Dentate Gyrus. Neuron, 93(3), 677-690.e5.

GoodSmith, D., Lee, H., Neunuebel, J. P., Song, H., \& Knierim, J. J. (2019). Dentate Gyrus Mossy Cells Share a Role in Pattern Separation with Dentate Granule Cells and Proximal CA3 Pyramidal Cells. The Journal of Neuroscience: The Official Journal of the Society for Neuroscience, 39(48), 95709584.

Gu, Y., Arruda-Carvalho, M., Wang, J., Janoschka, S. R., Josselyn, S. A., Frankland, P. W., \& Ge, S. (2012). Optical controlling reveals time-dependent roles for adult-born dentate granule cells. Nature Neuroscience, 15(12), 1700-1706.

Hainmueller, T., \& Bartos, M. (2018). Parallel emergence of stable and dynamic memory engrams in 
the hippocampus. Nature, 558(7709), 292-296.

Hainmueller, T., \& Bartos, M. (2020). Dentate gyrus circuits for encoding, retrieval and discrimination of episodic memories. Nature Reviews. Neuroscience, 21(3), 153-168.

Josselyn, S. A., \& Frankland, P. W. (2018). Memory Allocation: Mechanisms and Function. Annual Review of Neuroscience, 41, 389-413.

Josselyn, S. A., \& Tonegawa, S. (2020). Memory engrams: Recalling the past and imagining the future. Science, 367(6473). https://doi.org/10.1126/science.aaw4325

Khalaf, O., Resch, S., Dixsaut, L., Gorden, V., Glauser, L., \& Gräff, J. (2018). Reactivation of recallinduced neurons contributes to remote fear memory attenuation. Science, 360(6394), 12391242.

Kheirbek, M. A., Drew, L. J., Burghardt, N. S., Costantini, D. O., Tannenholz, L., Ahmari, S. E., Zeng, H., Fenton, A. A., \& Hen, R. (2013). Differential control of learning and anxiety along the dorsoventral axis of the dentate gyrus. Neuron, 77(5), 955-968.

Lacagnina, A. F., Brockway, E. T., Crovetti, C. R., Shue, F., McCarty, M. J., Sattler, K. P., Lim, S. C., Santos, S. L., Denny, C. A., \& Drew, M. R. (2019). Distinct hippocampal engrams control extinction and relapse of fear memory. Nature Neuroscience, 22(5), 753-761.

Lassalle, J. M., Bataille, T., \& Halley, H. (2000). Reversible inactivation of the hippocampal mossy fiber synapses in mice impairs spatial learning, but neither consolidation nor memory retrieval, in the Morris navigation task. Neurobiology of Learning and Memory, 73(3), 243-257.

Lee, I., \& Kesner, R. P. (2004). Encoding versus retrieval of spatial memory: double dissociation between the dentate gyrus and the perforant path inputs into CA3 in the dorsal hippocampus. Hippocampus, 14(1), 66-76.

Leutgeb, J. K., Leutgeb, S., Moser, M.-B., \& Moser, E. I. (2007). Pattern separation in the dentate gyrus and CA3 of the hippocampus. Science, 315(5814), 961-966.

Liu, X., Ramirez, S., Pang, P. T., Puryear, C. B., Govindarajan, A., Deisseroth, K., \& Tonegawa, S. (2012). Optogenetic stimulation of a hippocampal engram activates fear memory recall. Nature, 484(7394), 381-385.

Mahringer, D., Petersen, A. V., Fiser, A., Okuno, H., Bito, H., Perrier, J.-F., \& Keller, G. B. (2019). Expression of c-Fos and Arc in hippocampal region CA1 marks neurons that exhibit learningrelated activity changes. In bioRxiv (p. 644526). https://doi.org/10.1101/644526

Mau, W., Sullivan, D. W., Kinsky, N. R., Hasselmo, M. E., Howard, M. W., \& Eichenbaum, H. (2018). The Same Hippocampal CA1 Population Simultaneously Codes Temporal Information over Multiple Timescales. Current Biology: CB, 28(10), 1499-1508.e4.

McCauley, J. P., Petroccione, M. A., D’Brant, L. Y., Todd, G. C., Affinnih, N., Wisnoski, J. J., Zahid, S., Shree, S., Sousa, A. A., Migliore, R., Brazhe, A., Leapman, R. D., Khmaladze, A., Semyanov, A., Migliore, M., \& Scimemi, A. (2019). Circadian modulation of neurons and astrocytes controls synaptic plasticity in hippocampal area CA1. In bioRxiv (p. 666073). https://doi.org/10.1101/666073

Morellini, F. (2013). Spatial memory tasks in rodents: what do they model? Cell and Tissue Research, 354(1), 273-286.

NeuroElectro :: Dentate gyrus granule cell. (n.d.). Retrieved August 4, 2020, from https://neuroelectro.org/neuron/66/

Park, S., Kramer, E. E., Mercaldo, V., Rashid, A. J., Insel, N., Frankland, P. W., \& Josselyn, S. A. (2016). Neuronal Allocation to a Hippocampal Engram. Neuropsychopharmacology: Official Publication of the American College of Neuropsychopharmacology, 41(13), 2987-2993.

Perusini, J. N., Cajigas, S. A., Cohensedgh, O., Lim, S. C., Pavlova, I. P., Donaldson, Z. R., \& Denny, C. A. (2017). Optogenetic stimulation of dentate gyrus engrams restores memory in Alzheimer's disease mice. Hippocampus, 27(10), 1110-1122.

Pignatelli, M., Ryan, T. J., Roy, D. S., Lovett, C., Smith, L. M., Muralidhar, S., \& Tonegawa, S. (2019). Engram Cell Excitability State Determines the Efficacy of Memory Retrieval. Neuron, 101(2), 274-284.e5.

Poll, S., Mittag, M., Musacchio, F., Justus, L. C., Giovannetti, E. A., Steffen, J., Wagner, J., Zohren, L., 
Schoch, S., Schmidt, B., Jackson, W. S., Ehninger, D., \& Fuhrmann, M. (2020). Memory trace interference impairs recall in a mouse model of Alzheimer's disease. Nature Neuroscience. https://doi.org/10.1038/s41593-020-0652-4

Qin, H., Fu, L., Hu, B., Liao, X., Lu, J., He, W., Liang, S., Zhang, K., Li, R., Yao, J., Yan, J., Chen, H., Jia, H., Zott, B., Konnerth, A., \& Chen, X. (2018). A Visual-Cue-Dependent Memory Circuit for Place Navigation. Neuron, 99(1), 47-55.e4.

Ramirez, S., Liu, X., Lin, P.-A., Suh, J., Pignatelli, M., Redondo, R. L., Ryan, T. J., \& Tonegawa, S. (2013). Creating a false memory in the hippocampus. Science, 341(6144), 387-391.

Rangel, L. M., \& Eichenbaum, H. (2013). What's new is older. eLife, 2, e00605.

Rashid, A. J., Yan, C., Mercaldo, V., Hsiang, H.-L. L., Park, S., Cole, C. J., De Cristofaro, A., Yu, J., Ramakrishnan, C., Lee, S. Y., Deisseroth, K., Frankland, P. W., \& Josselyn, S. A. (2016). Competition between engrams influences fear memory formation and recall. Science, 353(6297), 383-387.

Reijmers, L. G., Perkins, B. L., Matsuo, N., \& Mayford, M. (2007). Localization of a stable neural correlate of associative memory. Science, 317(5842), 1230-1233.

Rennó-Costa, C., Lisman, J. E., \& Verschure, P. F. M. J. (2010). The mechanism of rate remapping in the dentate gyrus. Neuron, 68(6), 1051-1058.

Senzai, Y., \& Buzsáki, G. (2017). Physiological Properties and Behavioral Correlates of Hippocampal Granule Cells and Mossy Cells. Neuron, 93(3), 691-704.e5.

Tanaka, K. Z., He, H., Tomar, A., Niisato, K., Huang, A. J. Y., \& McHugh, T. J. (2018). The hippocampal engram maps experience but not place. Science, 361(6400), 392-397.

Trouche, S., Perestenko, P. V., van de Ven, G. M., Bratley, C. T., McNamara, C. G., Campo-Urriza, N., Black, S. L., Reijmers, L. G., \& Dupret, D. (2016). Recoding a cocaine-place memory engram to a neutral engram in the hippocampus. Nature Neuroscience, 19(4), 564-567.

van Dijk, M. T., \& Fenton, A. A. (2018). On How the Dentate Gyrus Contributes to Memory Discrimination. Neuron, 98(4), 832-845.e5.

Wiegert, J. S., Mahn, M., Prigge, M., Printz, Y., \& Yizhar, O. (2017). Silencing Neurons: Tools, Applications, and Experimental Constraints. Neuron, 95(3), 504-529.

Wietek, J., Beltramo, R., Scanziani, M., Hegemann, P., Oertner, T. G., \& Wiegert, J. S. (2015). An improved chloride-conducting channelrhodopsin for light-induced inhibition of neuronal activity in vivo. Scientific Reports, 5, 14807. 


\section{Methods.}

\section{Experimental animals}

c-Fos::tTA/cFos::shEGFP (TetTag) mice were obtained from the Jackson Laboratory and bred heterozygous crossed with C57BL6/J mice. Mice were group-housed with littermates until 2 weeks before AAV injections, then were single-caged and changed to doxycycline-containing food. Mice had access to food and water ad libitum and were kept in an animal facility next to the behavioral rooms on a reversed light-dark cycle (dark 7 am - 7 pm). All behavioral experiments were done at daytime during the dark phase of the cycle. Due to the weight of the implant and patch-cords (optogenetics), only male mice, between 20-60 weeks (>28g by the time of surgery) were included in the OptoWM experiments (Figure. 2-3). Both male and female mice were included in the cFos ensemble overlap experiments (Figure. 1, 4-6). All experiments were conducted in accordance with the German and European Community laws on protection of experimental animals and approved by the local authorities of the City of Hamburg (Behörde für Gesundheit und Verbraucherschutz, Lebensmittelsicherheit und Veterinärwesen, Nr. 100/15).

\section{Viral constructs}

pAAV-TREtight-iChloc-mKate was cloned from a backbone provided by Olaf Pongs. The iChloC sequence was taken from a CaMKIla-iChloC-2A-tDimer (Wietek et al., 2015) plasmid and linked to mKate for membrane fluorescent localization. iChloC-mKate was then cloned into the PAAV-TREtight promoter backbone. Virus production was done in the UKE Vector Facility packing the construct into an AAV 2/9 serotype.

\section{Surgical procedures}

To avoid hypothermia, a heating mat was placed under the animal throughout the whole surgery and under its cage for $1 \mathrm{~h}$ until full recovery. We provided post-surgery analgesia with Meloxicam mixed with softened Dox-food and left in a Petri dish in the cage for 3 days after surgery. Animals had at least 2 weeks for recovery before behavioral experiments.

AAV injection. TetTag mice were injected under analgesia and anesthesia using a stereotactic drill and injection robot (Neurostar). Mice were fixed to the frame under isoflurane anesthesia (1.5\%, mixed in 02 ), skin and connective tissue was removed and two craniotomies were performed using an automated drill on the desired coordinates. AAV9TRE-iChloC-mKate2 $\left(1 \times 10^{\wedge} 13 \mathrm{vg} / \mathrm{ml}\right)$ was delivered bilaterally into the dorsal dentate gyrus using a glass pipette attached to a $5 \mathrm{ml}$ Hamilton syringe. A single injection per site was 
performed using stereotaxic coordinates (-2.2 AP, $\pm 1.37 \mathrm{ML},-1.9 \mathrm{DV})$ with a volume of 500 $800 \mathrm{nl}$ on each side (injection speed: $100 \mathrm{nl} / \mathrm{min}$ ). After injection, the pipette was retracted $200 \mu \mathrm{m}$ and left for at least $5 \mathrm{~min}$ to avoid retroflux. After the injections, the bone surface was cleaned with $\mathrm{NaCl} 0.9 \%$ solution and skin was stitched.

Optic fiber implant. Mice in the optogenetic experiments were implanted with a bilateral optic fiber implant (Doric) targeting the DG sulcus (-2.2 AP, \pm 1.37 ML, $-1.5 \mathrm{DV}$ ) right after AAV injection. Implant was attached using C\&B dental cement onto the skull's surface and a protective cap was made out of an Eppendorf tube. The cap was secured then by applying acrylic resin (Pattern Resin LS, GC America) on all the exposed skull's surface in the implant surroundings.

\section{Doxycycline treatment}

Animals were given doxycycline-containing food (Altromin-Dox, $50 \mathrm{mg}$ per kg of body weight, red pellets). To tag the first cFos ensemble, animals were changed to regular doxycycline-free food (Altormin, light-brown pellets) $24 \mathrm{~h}$ before exposure to the desired task. To assure that the animals did not consume any Dox-food crumbles that had fallen into their cages, the bedding and cage were changed together with the food. The old nesting material was transferred to the new cage to decrease novelty-induced cFos expression. Dox food was given back exactly $24 \mathrm{~h}$ after removal, right before the behavioral task.

\section{Behavioral experiments}

All experiments were strictly timed due to cFos expression dynamics and temporal ensemble shift (Figure 4). For optogenetic experiments, mice were operated in batches of 6 , and tested weeks apart to perform the experiments at similar times of the day. For ensemble overlap experiments, mice were injected in batches of 10 and randomized to each of the groups. Mice that did not have adequate viral transfection, the implant location was off target, or performed poorly (floaters or implant intolerance) were not included in the analysis and therefore not every group has the same ' $n$ '. All experiments were recorded on digital video and automated mouse tracking was done with Ethovision XT 11.5.

\section{Water Maze (WM):}

Animals were handled by the experimenters (LA, PL) 1 week before the start of the pretraining sessions to reduce stress during behavioral tasks. Pre-training. Mice were pretrained for 2 days before ever visiting the WM arena (see below). Sessions ( $3 \times 60$ s trials on two days) were done in a small rectangular water tank in the dark, in the same room where 
the WM task was performed. Water level was $1 \mathrm{~cm}$ above the $10 \mathrm{~cm}$ diameter escape platform. The position of the platform was alternated between the left and right side of the tank between trials, keeping a distance of $5 \mathrm{~cm}$ from the walls to avoid thigmotaxis. Once the animals found the platform, a grid was presented until the animals climbed onto it and were returned to their home cages in the waiting area of the behavioral room. Training. The WM consisted of a circular tank (1.4 m diameter) with visual asymmetrical landmarks ${ }^{48}$, filled with water mixed with non-toxic white paint to prevent the animals from seeing the platform (submerged by $1 \mathrm{~cm}$ ). The platform was placed in the center of the east quadrant during regular training and switched to the opposite (west) quadrant for reversal training (max. training length: $90 \mathrm{~s}$ ). To test spatial reference memory, a probe trial without a platform was performed on each day. Mice underwent six trials every day (4 training trials (TT, $90 \mathrm{~s})+1$ probe trial (PT, $60 \mathrm{~s})+1 \mathrm{TT}$ ), inter-trial interval (ITI) was 8-10 s. For the TTs, mice were lowered into the tank facing the wall in different, pseudo-randomized positions (avoiding the target quadrant). In the PT, mice were lowered in the center of the tank. An opaque cup-sized chamber attached to a pole was used to transfer the mice from their home cage to the drop position and a plastic grid attached to a pole was used to pick up the mice. Mice were picked up $10 \mathrm{~s}$ after they found the platform and were returned to their home cage. Mice that did not find the platform during the length of the $\mathrm{TT}$, were guided to the platform using the grid and were picked up after a $10 \mathrm{~s}$ on-platform waiting period. For both pre-training and WM, water temperature was $19^{\circ} \mathrm{C}$ and a heating lamp was placed on the waiting area to avoid hypothermia.

\section{Home caged and open field control experiments:}

To evaluate the temporal effect of cFos ensembles, mice were unperturbed in their home cage $(\mathrm{HC})$ during the off-dox period but no behavior was analysed. Mice were either sacrificed right after $24 \mathrm{~h}$ from Dox removal ( $0 \Delta$ group) or put back on Dox and sacrificed after 6 or $24 \mathrm{~h}$. Both cFos+ tagging events were designed to have the same amount of trials and training length in all experiments. However, for the open field experiment, mice were placed inside a square arena ( $50 \times 50 \mathrm{~cm}, 50 \mathrm{lux}$ ) for $20 \mathrm{~min}$ but were kept in the behavioral room the same time as their WM counterparts.

\section{Optogenetic inhibition in the water maze:}

Mice were connected to two ultra-thin ( $200 \mu \mathrm{m}$ core diameter, $2 \mathrm{~m}$, Doric) optic fibers using ceramic ferrules (Doric Lenses, Canada) and put back to their home cage for at least 5 min before any behavioral task. In pre-training trials ( 2 days, 3 trials per day), mice were connected and disconnected before every trial to habituate them to the tether. During 
training, only one mouse was tethered at a time so trials were not intercalated. Instead, one mouse was trained from beginning to end before the next one. This allowed training of only 6 mice per day. To avoid stress-related cFos tagging, mice were never tethered during the off-Dox period. From day 1-5, mice only received light during two PTs in a counterbalanced light ON/OFF fashion (day $3 \& 5$, Figure. 2). On day 6, only mice in the RT-S receive light during the reversal TTs (rTT), but not during PTs. Mice in the RT-C did not receive light on day 6 (Figure. 3). Training protocol. Day 1 (Off-Dox): no tether, 4TT + 1 PT + 1TT; Day 2: tether, 3TT + 1PT + 1TT; Day 3: 2PTs tethered + 3TTs without tether; Day 4: 4TT + 1PT + 1TT(tethered); Day 5: tethered, 2TT + 2PTs; Day 6: tethered, 4 rTTs + $1 \mathrm{PT}$ ). In all trials with tether, the weight of the fibers was compensated with a white helium balloon $(0.07 \mathrm{~N}$ pull force), attached to a light fiber $\sim 30 \mathrm{~cm}$ above the mouse with a transparent fishing line long enough to keep the balloon out of the field of view of the video camera. The I.T.I. was 10-15 min. Mice were brought back to their $\mathrm{HC}$ after each trial but were not untethered. The HC was placed close to the WM tank but behind a curtain on a mobile platform to alternate the exit location in a pseudo-random fashion. Blue light $(473 \mathrm{~nm})$ was delivered using a laser combiner (LightHUB, Omicron) connected to a commutator (FRJ_1x2i_FC-2FC_0.22 Doric Lenses, Canada) to split the output into two fibers. The commutator was placed close to the ceiling, at the same height as the camera. To ensure inhibition before and after mice were in the tank, light was delivered in 20 ms pulses repeated at $1 \mathrm{~Hz}$ during $120 \mathrm{~s}$ for TTs and rTTs (90 s). For PTs (60 s), light was delivered for $90 \mathrm{~s}$. Mice were always sacrificed $1.5 \mathrm{~h}$ after the last trial.

Ex vivo brain processing and cFos immunofluorescence (IF) staining

Mice from all behavioral groups were sacrificed $3.5 \mathrm{~h}$ after the last trial for shEGFP IF staining (1.5 $\mathrm{h}$ after for cFos IF staining) by ketamine/xylazine (100/10 mg kg-1) intraperitoneal injection and intracardially perfused with $1 x$ phosphate-buffered saline (PBS, Sigma) followed by $4 \%$ paraformaldehyde (PFA, Roth). Brains were extracted and stored in $4 \%$ PFA for at least $24 \mathrm{~h}$ at $4^{\circ} \mathrm{C}$. Before sectioning, brains were washed with 1 xPBS for $20 \mathrm{~min}$ at room temperature (RT). The dorsal hippocampal region (-1.2 to -2.3 AP from bregma) was cut in $40 \mu \mathrm{m}$ coronal sections using a vibratome (Leica VT100S) and collected in PBS. From each series, six sections were selected from Bregma -1.7 to $-2.3 \mathrm{~mm}$ and incubated in blocking buffer (1x PBS, 0,3\% TritonX, 5\% goat serum) for $2 \mathrm{~h}$ at RT. Next, sections were placed in the primary antibody carrier solution (1X PBS, 0,3\% TritonX, 1\% goat serum, 1\% $\mathrm{BSA}$ ) and incubated overnight at $4^{\circ} \mathrm{C}$. After $3 \mathrm{x}$ washing with $1 \mathrm{xPBS}$ for $5 \mathrm{~min}$, the sections were incubated for $2 \mathrm{~h}$ at RT in the secondary antibody carrier solution (1x PBS, 0,3\% TritonX, 5\% goat serum). Sections were washed $3 \times 10$ min in $1 \times$ PBS, stained with 4',6- 
diamidino-2-phenylindole (DAPI, 1:1000) for 5 min and mounted on coverslips using ImmuMount (Shandon). Primary antibodies: Chicken Anti GFP polyclonal antibody, Invitrogen (A10262, Lot 1972783); Rabbit Anti-tRFP (mKate2) antibody, Evrogen (AB233, Lot 23301040466), Rabbit anti cFos, Synaptic Systems (226003, Lot ). Secondary antibodies: Alexa Fluor 488 conjugated secondary antibody (goat anti-chicken, 1:1000, Life technologies; A11039; Alexa Fluor 568 conjugated secondary antibody (goat anti-rabbit, 1:1000, Life Technologies, A11011).

\section{Confocal imaging}

Before imaging, mounted slides were labeled with a code to blind the analyst. The stained slices were imaged with a confocal microscope (Olympus Fluoview FV 1000) using a 20x oil immersion objective (UPLSAPO 20X NA: 0.85). From each of the 6 sections per brain, a stack of 6 images (step size $2.5 \mu \mathrm{m}$ ) from both dentate gyri was taken. 3 channel images were obtained at a $1024 \times 1024$ resolution. Excitation/emission filters were selected using the dye selection function of the Olympus software (Alexa 405 (DAPI), Alexa 488 (shEGFP) and Alexa 568 (mKate)). The image acquisition settings were optimized once and kept constant for all images within an experimental data set. Images were not deconvolved, gamma-adjusted or filtered. Example images presented in figures, the look-up-table was linearly adjusted, filtered with a 2-pixel median filter and cropped to show relevant regions.

\section{Ensemble size calculation and overlap analysis}

Confocal image stacks (green: shEGFP; pseudo-colored magenta: mKate; blue: DAPI) were analyzed using Imaris (Bitplane). The DAPI channel was used to create a surface of the upper and lower blade of DG (granule cell layer, GCL). The volume of the GCL was used to estimate the total GC number based on published cell densities ${ }^{49}$ and to mask the green and the pseudo-colored magenta channel to restrict analysis to the GCL. Automatic spot detection was used to identify shEGFP+ cells in the green channel. The quality filter (round nuclei with a diameter of $8 \mu \mathrm{m}$ ) was once adjusted by the analyst to include weakly expressing cells and then applied to every image stack of the same experiment. False positive spots (e.g. staining artifacts) were manually removed. It was not possible to detect mKate-expressing GCs automatically, as only the plasma membrane was labeled. To count mKate-positive cells, spots were placed manually (spot size $10.6 \mu \mathrm{m}$ ) using the pseudo-colored magenta channel only. Double-positive cells (distance between shEGFP+ and mKate+ spots $<5 \mu \mathrm{m}$ ) were identified using a Matlab script. They were then manually inspected to check for artifacts and, if necessary, corrected. From the Imaris analysis, we calculated the following values using Excel spreadsheets: 


$$
\text { Number of granule cells }=\frac{\text { DAPI surface volume }}{\text { reported GC density }}
$$

fraction of shEGFP tagged cells $(\%)=\frac{\text { number of shEGFP }+ \text { cells }}{\text { number of } G C s}$

fraction of mKate tagged cells $(\%)=\frac{\text { number of } m \text { Kate } 2+\text { cells }}{\text { number of } G C s}$

fraction double tagged cells $(\%)=\frac{\text { number of double positive cells }}{\text { number of GCS }}$

expected reactivation $=$ fraction of shEGFP cells $*$ fraction of $m$ Kate cells

$$
\text { Reactivation above chance }=\frac{\text { number of double positive cells }}{\text { expected reactivation }}
$$

Preparation of hippocampal slice cultures

Hippocampal slice cultures from TetTag mice or Wistar rats were prepared at postnatal day 8 or 4-7 respectively as described(Gee et al., 2017). Briefly, animals were anesthetized with $80 \%$ $\mathrm{CO}_{2} 20 \% \mathrm{O}_{2}$ and decapitated. Hippocampi were dissected in cold slice culture dissection medium containing (in $\mathrm{mM}$ ): 248 sucrose, $26 \mathrm{NaHCO}_{3}, 10$ glucose, $4 \mathrm{KCl}, 5 \mathrm{MgCl}_{2}, 1 \mathrm{CaCl}_{2}, 2$ kynurenic acid, $0.001 \%$ phenol red (310-320 mOsm kg-1 saturated with $95 \% \mathrm{O}_{2}, 5 \% \mathrm{CO}_{2}, \mathrm{pH}$ 7.4). Tissue was cut into $400 \mu \mathrm{M}$ thick sections on a tissue chopper and cultured at the medium/air interface on membranes (Millipore PICMORG50) at $37{ }^{\circ} \mathrm{C}$ in $5 \% \mathrm{CO}_{2}$. No antibiotics were added to the slice culture medium which was partially exchanged (60-70\%) twice per week and contained (for $500 \mathrm{ml}$ ): $394 \mathrm{ml}$ Minimal Essential Medium (Sigma M7278), $100 \mathrm{ml}$ heat inactivated donor horse serum (H1138 Sigma), 1 mM L-glutamine (Gibco 25030-024), $0.01 \mathrm{mg} \mathrm{ml}^{-1}$ insulin (Sigma I6634), $1.45 \mathrm{ml} 5 \mathrm{M} \mathrm{NaCl}$ (S5150 Sigma)), 2mM MgSO 4 (Fluka 63126), $1.44 \mathrm{mM} \mathrm{CaCl}_{2}$ (Fluka 21114), 0.00125\% ascorbic acid (Fluka 11140), 13 mM D-glucose (Fluka 49152).

Calibration of iChloC in dentate granule cells of organotypic cultures

Organotypic rat slice cultures were infected with AAV2/9 pAAV-CaMKII-ChR (E83Q, E90R, E101S, D156N, T159C)-2A-tdimer2 (iChloC) 81-2 (Virus titer: 3.75×1012) after 28 days in vitro (DIV). At DIV 36, patch clamp recordings of dentate granule cells were performed using a BX51WI microscope (Olympus), a Multiclamp 700B amplifier (Molecular Devices), a motorized micromanipulator MP-285 (Sutter Instruments) and Wavesurfer software programmed with Matlab (The MathWorks). Slices were placed in HEPES-buffered solution (21-23 ${ }^{\circ} \mathrm{C}$ ) consisted 
of (in mM): $145 \mathrm{NaCl}, 10 \mathrm{Na}$-HEPES, $12.5 \mathrm{D}$-glucose, $2.5 \mathrm{KCl}, 2 \mathrm{CaCl}_{2}, 1 \mathrm{MgCl}_{2}, 1.25 \mathrm{NaH}_{2} \mathrm{PO}_{4}$ (pH 7.4). Patch pipettes with a tip resistance of 3-5 $\mathrm{M} \Omega$ were filled with intracellular solution (in mM): $135 \mathrm{~K}$-gluconate, $4 \mathrm{MgCl}_{2}, 4 \mathrm{Na}_{2}$-ATP, $0.4 \mathrm{Na}$-GTP, $10 \mathrm{Na}_{2}$-phosphocreatine, 3 sodiumL-ascorbate and 10 HEPES (pH 7.2). A LED light engine (SpectraX, Lumencor) was used for delivery of light pulses (476 $\mathrm{nm}$ ) through a 60x (Olympus) objective. Light intensity was measured with a calibrated power meter (LaserCheck, Coherent) and divided by the illuminated field of view $\left(0.169 \mathrm{~mm}^{2}\right)$ to achieve $10 \mathrm{~mW} / \mathrm{mm}^{2}$. The AP threshold was determined by injecting current steps from 0 - 150 pA. Patched-clamped cells were stimulated every 5 s with a 20 ms 150 pA square pulse for 60 seconds. 20 ms before stimulation 4, 7, or 10 light pulses ( $20 \mathrm{~ms}, 476 \mathrm{~nm}, 10 \mathrm{~mW} / \mathrm{mm}^{2}$ ) were applied. Series resistance was between 10 and $40 \mathrm{M} \Omega$.

\section{Statistical analysis}

The definition and the exact value for $\mathrm{n}$ is given in the text or in figure legends. Replication of experiments is reported in figure legends. Statistical analysis was performed using GraphPad Prism (v8). 